ARTICLE

https://doi.org/10.1038/s41467-019-13809-8

\title{
Ketamine disinhibits dendrites and enhances calcium signals in prefrontal dendritic spines
}

\author{
Farhan Ali', Danielle M. Gerhard', Katherine Sweasy', Santosh Pothula', Christopher Pittenger (1) 1,2, \\ Ronald S. Duman ${ }^{1,3}$ \& Alex C. Kwan (1) ${ }^{1,3 *}$
}

A subanesthetic dose of ketamine causes acute psychotomimetic symptoms and sustained antidepressant effects. In prefrontal cortex, the prevailing disinhibition hypothesis posits that $\mathrm{N}$-methyl-d-aspartate receptor (NMDAR) antagonists such as ketamine act preferentially on GABAergic neurons. However, cortical interneurons are heterogeneous. In particular, somatostatin-expressing (SST) interneurons selectively inhibit dendrites and regulate synaptic inputs, yet their response to systemic NMDAR antagonism is unknown. Here, we report that ketamine acutely suppresses the activity of SST interneurons in the medial prefrontal cortex of the awake mouse. The deficient dendritic inhibition leads to greater synaptically evoked calcium transients in the apical dendritic spines of pyramidal neurons. By manipulating NMDAR signaling via GluN2B knockdown, we show that ketamine's actions on the dendritic inhibitory mechanism has ramifications for frontal cortex-dependent behaviors and cortico-cortical connectivity. Collectively, these results demonstrate dendritic disinhibition and elevated calcium levels in dendritic spines as important local-circuit alterations driven by the administration of subanesthetic ketamine.

\footnotetext{
${ }^{1}$ Department of Psychiatry, Yale University School of Medicine, New Haven, CT 06511, USA. ${ }^{2}$ Child Study Center, Yale University School of Medicine, New Haven, CT 06511, USA. ${ }^{3}$ Department of Neuroscience, Yale University School of Medicine, New Haven, CT 06511, USA. *email: alex.kwan@yale.edu
} 
K etamine is a non-competitive $\mathrm{N}$-methyl-d-aspartate receptor (NMDAR) antagonist. In humans, systemic administration of ketamine at a subanesthetic dose have both acute and sustained effects. Acutely, within about $1 \mathrm{~h}$ following administration, subjects experience psychotomimetic symptoms, perceptual aberrations, and cognitive impairments ${ }^{1}$. Because the acute effects mimic the positive and negative symptoms of schizophrenia, ketamine has found use as a tool for studying glutamatergic dysfunction and evaluating antipsychotic compounds $^{2,3}$. On a longer timescale, starting from 2 to $4 \mathrm{~h}$ following administration, patients suffering from major depressive episodes report a robust antidepressant effect that remain for about a week ${ }^{4,5}$. Although these behavioral effects are well established and have substantial translational implications, the mechanisms by which ketamine impacts neural activity remain unclear.

In rodents, intraperitoneal or subcutaneous injection of subanesthetic ketamine activates multiple, distributed brain regions, as indicated by brain-wide mapping of metabolic activity ${ }^{6}$. Among the regions with elevated metabolic activity is the medial prefrontal cortex, including the ventral prelimbic and infralimbic sub-regions, as well as the dorsal cingulate and secondary motor sub-regions (Cg1/M2). This locus is consistent with earlier works demonstrating that, for NMDAR antagonists, there is a dosedependent increase in the extracellular levels of glutamate in the rat prefrontal cortex ${ }^{7}$, as well as an increase in the overall firing rate of prefrontal cortical neurons ${ }^{8}$. Since NMDAR is an ionotropic glutamate receptor that contributes to postsynaptic depolarization, why would an antagonist lead to more spiking activity? One prevailing idea is the disinhibition hypothesis, which posits that NMDAR antagonists at low dose may act preferentially on GABAergic neurons, thereby disinhibiting excitatory neurons ${ }^{9,10}$. In support of the disinhibition hypothesis, fast-spiking GABAergic neurons, identified based on their narrow spike waveforms, exhibit a concomitant reduction in firing in vivo at the time of prefrontal hyperactivity during NMDAR hypofunction ${ }^{11}$.

However, GABAergic neurons are not homogeneous. Fastspiking cells constitute a fraction of the GABAergic neurons, and another non-overlapping subpopulation is the somatostatinexpressing (SST) interneurons. SST interneurons are a major subpopulation that constitutes $20 \%$ of the GABAergic neurons in the mouse frontal cortex ${ }^{12}$. Unlike the fast-spiking cells which inhibit cell bodies, SST interneurons include the so-called Martinotti cells that selectively target apical dendrites and thus are poised to control synaptic inputs and dendritic integration ${ }^{13}$. The cell-type-specific differences suggest that if SST interneurons were altered, then the resulting circuit-level and behavioral impacts may be quite distinct from those involving perturbations of fastspiking cells. Still unknown, however, is whether and how SST interneurons and dendritic inhibition may be impacted by subanesthetic ketamine.

In this study, we use two-photon calcium imaging to characterize SST interneurons, SST axons, pyramidal neurons, and pyramidal dendritic spines in awake, head-fixed mice. For each cellular compartment, we compare spontaneous calcium transients before and within an hour after the administration of subanesthetic ketamine, relative to saline control. We find that ketamine suppresses the activity of SST interneurons in the medial prefrontal cortex. We further show that this loss of dendritic inhibition contributes to elevated synaptic calcium transients in the apical dendritic spines of pyramidal neurons. Based on these results, we conclude that the dendritic disinhibition and calcium elevations in dendritic spines are important components of ketamine's actions on the prefrontal cortex.

\section{Results}

Cell-type-specific responses to subanesthetic ketamine. We performed two-photon microscopy on awake, head-fixed mice (Fig. 1a), targeting the Cg1/M2 sub-regions of the medial prefrontal cortex (Fig. 1b). Initially, while mice were head-fixed under the two-photon microscope, we recorded body motion using an infrared camera. This was because systemic administration of subanesthetic ketamine induces hyperlocomotion in rodents ${ }^{14}$, and we wanted to avoid movement as a confound in our imaging experiments. We observed that ketamine $(10 \mathrm{mg} / \mathrm{kg}$, s.c.) increased body motion but only transiently, and therefore limited all of our data collection to 30-60 min post-injection (Fig. 1c, d). For calcium imaging, we used AAV1-CamKIIGCaMP6f-WPRE-SV40 to express the calcium-sensitive fluorescent protein GCaMP6f in pyramidal neurons in $\mathrm{Cg} 1 / \mathrm{M} 2$, and imaged spontaneous fluorescence transients from the awake mouse (Fig. 1e). From the fluorescence transients, we detected calcium events using a peeling method based on template matching ${ }^{15}$. Ketamine $(10 \mathrm{mg} / \mathrm{kg})$ increased the rate of spontaneous calcium events in pyramidal neuron cell bodies in layer $2 / 3$ (200-400 $\mu \mathrm{m}$ from the dura) (ketamine: $23.7 \pm 2.1 \%$, saline: $9.4 \pm$ $1.9 \%$, relative to pre-injection, mean \pm s.e.m.; $P=3 \times 10^{-8}$, twosample $t$-test; Fig. 1f, g; Supplementary Fig. 1a). The elevation in calcium event rates did not correlate with animal movement (Supplementary Fig. 1b) and was consistent across individual mice (Supplementary Fig. 2a). Ketamine's effect lasted up to $\sim 50$ min post-injection (Supplementary Fig. 3a). Somatic calcium transients have been shown to directly relate to the firing rate of cortical neurons ${ }^{16,17}$, therefore the elevated calcium event rates reflect hyperactivity of pyramidal neurons, consistent with previous reports ${ }^{11,18}$. To characterize the effect of ketamine on the SST subpopulation of GABAergic neurons, we injected AAV1Syn-DIO-GCaMP6s-WPRE-SV40 into Cg1/M2 of SST-IRES-Cre mice to express GCaMP6s selectively in SST interneurons (Fig. 1h). Following ketamine, the rate of spontaneous calcium events was reduced for the cell bodies of layer 2/3 SST interneurons (ketamine: $-13 \pm 3 \%$, saline: $13 \pm 6 \%$, relative to preinjection, mean \pm s.e.m.; $P=1 \times 10^{-4}$, two-sample $t$-test; Fig. $1 \mathrm{i}$, j, Supplementary Fig. 1c, d; Supplementary Fig. 2b; Supplementary Fig. 3b). These results indicate that ketamine increases the activity of pyramidal neurons while suppressing the activity of SST GABAergic neurons.

Ketamine disinhibits dendrites and raises calcium in spines. SST interneurons inhibit selectively dendrites including the dendritic spines of pyramidal neurons ${ }^{13}$. The ketamine-induced reduction of SST interneuron activity is therefore expected to diminish dendritic inhibition. To determine if this may apply to the apical dendritic compartment, we imaged the inhibitory inputs-SST axonal boutons in the superficial layer of Cg1/M2 $(<200 \mu \mathrm{m}$ from the dura) (Fig. 2a), which showed fewer calcium events after ketamine $(10 \mathrm{mg} / \mathrm{kg}$ ) (Fig. 2b; Supplementary Fig. 2c; Supplementary Fig. 3c). We also imaged directly the postsynaptic calcium signals in the apical dendrite tufts of pyramidal neurons. We focused on the dendritic spines (Fig. 2c), because subthreshold synaptic activation can be characterized by their accompanying calcium elevations in the spine compartment with minimal outflow to the dendritic shaft ${ }^{19}$. To restrict our analyses to these localized fluorescence transients, we used a standard procedure to regress out the contribution from the dendritic shaft ${ }^{17,19}$, following which we applied the same calcium event detection procedure. Ketamine $(10 \mathrm{mg} / \mathrm{kg})$ induced a $43.42 \pm$ $0.01 \%$ increase in spontaneous calcium event rate for dendritic spines in Cg1/M2, significantly higher than saline controls (4.34 \pm $0.01 \%, P=0.02$, two-sample $t$-test; Fig. 2 c, d; Supplementary 
a

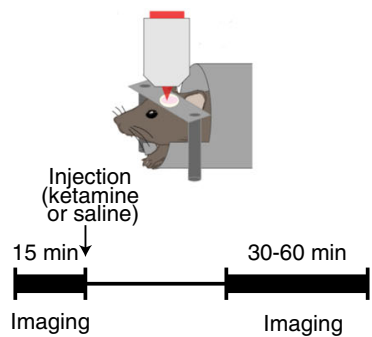

b

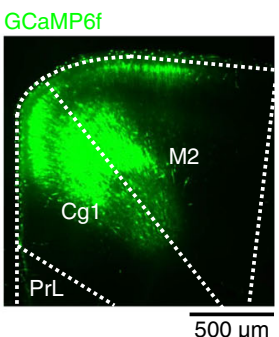

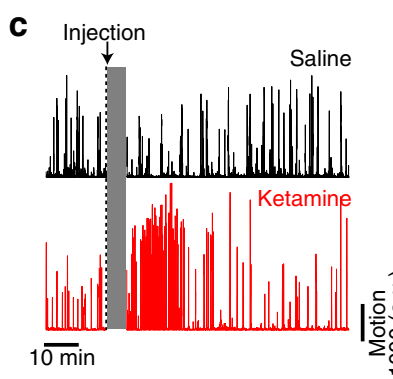
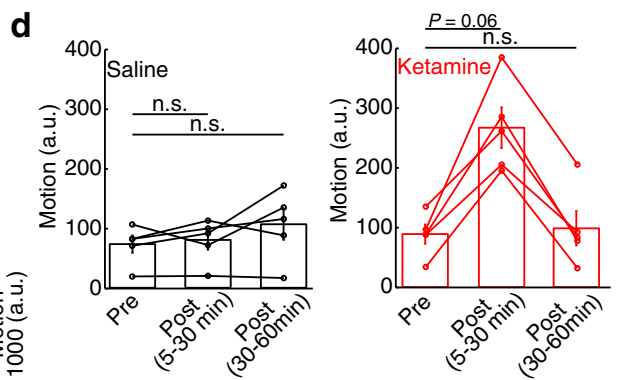

e
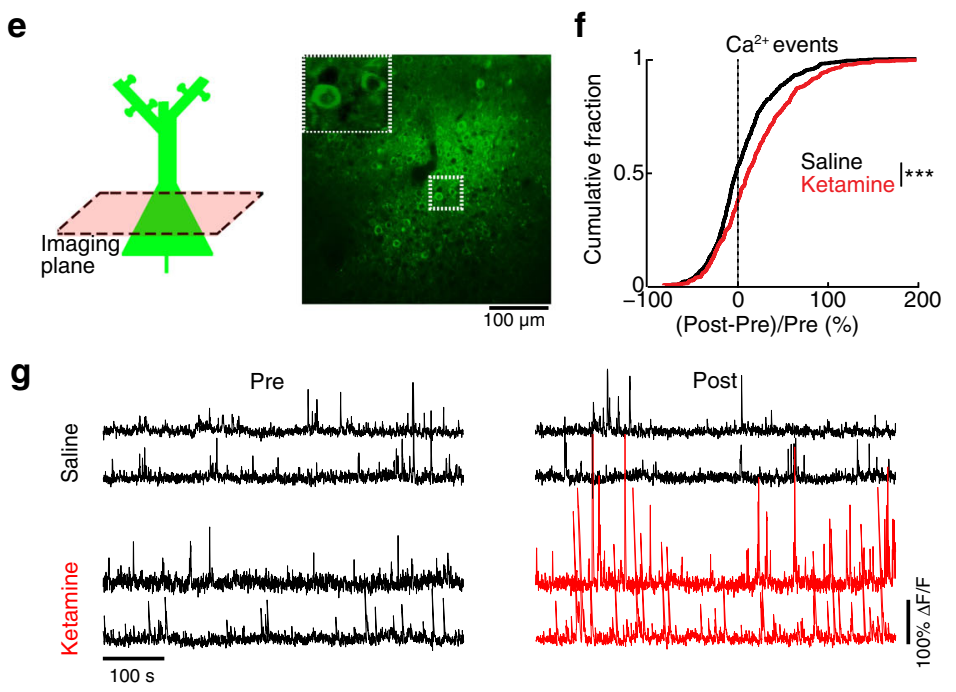

h


j

Pre
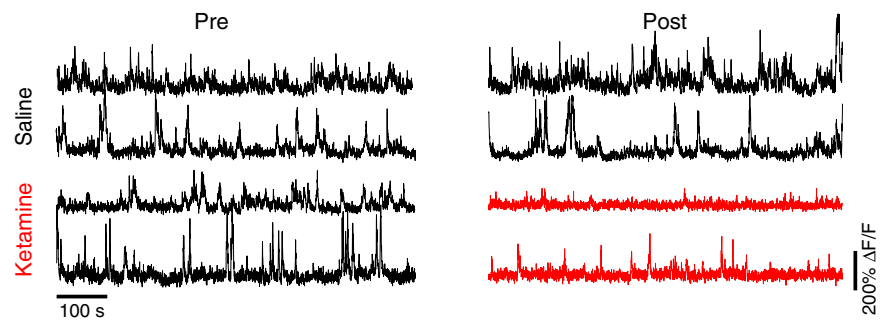

Fig. 2d; Supplementary Fig. 3d). To characterize the temporal dynamics of the aberrant transients, we identified calcium events that co-occurred in each image frame, thereby separating amplitude (number of events in each frame) from frequency (number of frames with at least one event). Both the amplitude

and frequency parameters were elevated following ketamine (Fig. 2e). The results did not depend on the specifics of the template in the event detection procedure, because an alternative analysis based on threshold crossing yielded similar conclusions (Supplementary Fig. 4). Interestingly, the ketamine-induced 
Fig. 1 Effects of subanesthetic ketamine on the activity of pyramidal neurons and SST interneurons in vivo. a Schematic and timeline of the experiments. b Coronal histological section, showing the extent of AAV-mediated expression of GCaMP6f in the mouse medial prefrontal cortex. Cg1, cingulate cortex. M2, secondary motor cortex. PrL, prelimbic cortex. c Example motion traces from a head-fixed animal during a two-photon imaging session before and after saline injection (black), and another session before and after ketamine $(10 \mathrm{mg} / \mathrm{kg}$ ) injection (red). Gray shading denotes the $5 \mathrm{~min}$ during which the injection was made and imaging was done to verify that the field of view had not shifted. $\mathbf{d}$ Time-averaged motion (mean \pm s.e.m.) for epochs including preinjection, 5-30 min post-injection, and 30-60 min post-injection for saline (left) and ketamine (right). For saline injection (black), there were no detectable differences between epochs (pre-injection vs. 5-30 min post-injection: $P=0.6$; pre-injection vs. 30-60 min post-injection: $P=0.1$; Wilcoxon signed rank test). For ketamine (red), hyperlocomotion was detected transiently following injection (pre-injection vs. 5-30 min post-injection: $P=0.06$; pre-injection vs. 30-60 min post-injection: $P=1$; Wilcoxon signed rank test). Each point is an imaging session. $n=5$ animals each for saline and ketamine. e Schematic of imaging location, and an in vivo two-photon image of GCaMP6s-expressing pyramidal neurons in Cg1/M2. Inset, magnified view of neuronal cell bodies. f The normalized difference in the rate of spontaneous calcium events of pyramidal neurons. Normalized difference was calculated as post-injection minus pre-injection values normalized by the pre-injection value (ketamine $\left(10 \mathrm{mg} / \mathrm{kg}\right.$ ): $23.7 \pm 2.1 \%$, saline: $9.4 \pm 1.9 \%$, mean \pm s.e.m.; $P=3 \times 10^{-8}$, two-sample $t$-test). For ketamine, $n=613$ cells from 5 animals. For saline, $n=681$ cells from 5 animals. $\mathbf{g}$ Each row shows time-lapse fluorescence transients from the same pyramidal cell in the pre-injectiion (left) and post-injection (right) periods. Two example cells were plotted for saline injection (black), and two other examples were plotted for ketamine $(10 \mathrm{mg} / \mathrm{kg}$ ) injection (pre-injection: black; post-injection: red). h-j Same as (e-g) for GCaMP6s-expressing SST interneurons in Cg1/M2 of SST-IRES-Cre animals (ketamine (10 mg/kg): $-12 \pm 3 \%$, saline: $13 \pm 6 \%$, mean \pm s.e.m.; $P=1 \times 10^{-4}$, two-sample $t$-test). For ketamine, $n=198$ cells from 5 animals. For saline, $n=179$ cells from 5 animals. ${ }^{\star} P<0.05 ;{ }^{\star \star} P<0.01 ;{ }^{\star \star \star} P<0.001$; n.s., not significant.

elevation of spine calcium transients was regionally specific, because there was little effect when we repeated the experiment in the primary motor cortex (Fig. 3; Supplementary Fig. 2e). Altogether, the results demonstrate that ketamine disinhibits apical dendrites, leading to elevated calcium transients in the apical dendritic spines of prefrontal pyramidal neurons.

Elevated synaptic calcium responses to long-range inputs. By focusing on fluorescence transients that are localized to dendritic spines, we are characterizing calcium signals related to synaptic activation. It follows then that a more stringent and explicit test for a synaptically activated signal to directly stimulate the afferent inputs. To test this, we take advantage of the knowledge that apical dendritic spines receive long-range cortico-cortical inputs $^{20}$, and $\mathrm{Cg} 1 / \mathrm{M} 2$ receives an abundance of monosynaptic excitatory inputs from the retrosplenial cortex (RSC). We implanted electrodes to stimulate RSC (biphasic pulse with duration of $10 \mathrm{~ms}$ and a peak amplitude of $\pm 150 \mu \mathrm{A}$ ), and simultaneously imaged the evoked calcium responses in Cg1/M2 (Fig. 4a). First, we confirmed that electrical stimulation activated the long-range inputs, by imaging the axons emanating from RSC in $\mathrm{Cg} 1 / \mathrm{M} 2$ and finding stimulation-aligned calcium transients (Supplementary Fig. 5a-e). Then, in a separate group of animals, we imaged the evoked calcium responses in single dendritic spines in $\mathrm{Cg} 1 / \mathrm{M} 2$ while varying the stimulation strength (number of pulses $=1,2,4,8,16,32$, or 64 per trial; Fig. 4b). Ketamine $(10 \mathrm{mg} / \mathrm{kg})$, but not saline, increased evoked responses in Cg1/M2 dendritic spines (ketamine: $\mathrm{F}(6,1146)=6.6, P=7 \times 10^{-7}$, saline: $\mathrm{F}(6,1218)=20.0, P=0.06$, two-way interaction between time (pre vs. post) and stimulation strength, two-way mixed ANOVA after three-way mixed ANOVA; Fig. 4c, d). Across all imaged spines, the increase in evoked calcium response was more pronounced at higher stimulation strengths (Fig. 4d; Supplementary Fig. 5f-g). By directly controlling the activation of long-range inputs, these results provide evidence that ketamine elevates the synaptically evoked component of calcium transients in prefrontal dendritic spines.

Ketamine's effect on dendrites rely on GluN2B in SST neurons. We wanted to determine the causal relations between ketamine, SST interneuron activity, and synaptic calcium signals. We predict that if ketamine exerts its effects through SST interneurons, then knocking down NMDAR signaling in SST interneurons should reproduce the local-circuit alterations and render additional ketamine ineffective. To test this prediction, we created AAV1-CMV-dsRed-pSico-GluN2BshRNA to induce Credependent expression of short hairpin RNA (shRNA) against GluN2B (Fig. 5a) 21,22. We targeted the GluN2B subunit because neocortical SST interneurons contain abundant GluN2B transcripts $^{23,24}$, and the subunit contributes a sizable fraction of NMDAR-mediated current in the related low-threshold spiking interneurons in the rodent medial prefrontal cortex ${ }^{25}$. This virus was confirmed to reduce GluN2B expression in a Cre-dependent manner in vitro $(-42 \pm 3 \%$ relative to controls; Supplementary Fig. 6) and to decrease NMDAR-mediated currents in brain slices $^{26}$. Injected into Cg1/M2 of SST-IRES-Cre animals (Fig. 5b), viral-mediated knockdown of GluN2B in SST interneurons (GluN2B-SST KD) reduced SST interneuron activity at baseline (GluN2B-SST KD: $1.5 \pm 0.2 \mathrm{~Hz}$, controls: $1.9 \pm 0.1 \mathrm{~Hz}$, mean \pm s.e. m.; $P=0.04$, two-sample $t$-test; Fig. $5 \mathrm{c}$ ), mimicking the effects of ketamine. Notably, GluN2B-SST KD blocked further ketamineinduced $(10 \mathrm{mg} / \mathrm{kg})$ influence on SST interneuron activity (Fig. 5d). In a separate group of animals, we imaged dendritic spines, finding that GluN2B-SST KD increased the rate of spontaneous calcium events at baseline (GluN2B-SST KD: $0.85 \pm$ $0.03 \mathrm{~Hz}$, controls: $0.73 \pm 0.04 \mathrm{~Hz} ; P=0.01$, two-sample $t$-test; Fig. 5e), and additional ketamine $(10 \mathrm{mg} / \mathrm{kg})$ had no detectable effect (Fig. 5f). The causal manipulations thus confirm the predicted role of SST interneurons and highlight the involvement of GluN2B in mediating ketamine's effect on the dendritic spines.

Behavioral consequence of downregulating SST neuron activity. Ketamine exerts numerous acute behavioral effects, so next we asked to what extent dendritic disinhibition in the medial prefrontal cortex may be involved. We tested animals on three behavioral assays: trace fear conditioning, pre-pulse inhibition and locomotor activity, and determined the effect of ketamine with or without bilateral GluN2B-SST KD (Fig. 6a). In trace fear conditioning, an auditory stimulus is paired with a footshock with an intervening trace period. This assay requires associative learning across a temporal gap, which relies on the medial prefrontal cortex ${ }^{27}$. SST-IRES-Cre animals received ketamine $(10 \mathrm{mg} / \mathrm{kg}) 15 \mathrm{~min}$ before conditioning, and subsequently showed impaired freezing $24 \mathrm{~h}$ later in the 15 -s trace but not in the delay (no trace) paradigm (Fig. 6b), indicating an impairment specific to temporal association but not learning in general. After bilateral GluN2B-SST KD in Cg1/M2, ketamine no longer had detectable effects on trace fear learning (Fig. 6b). Pre-pulse inhibition, a cross-species measure of sensorimotor gating, is regulated by prefrontal cortical activity ${ }^{28}$. Ketamine $(40 \mathrm{mg} / \mathrm{kg})$ reduced prepulse inhibition in control SST-IRES-Cre animals (main effects of 
a

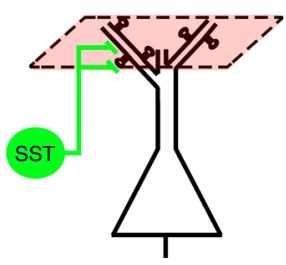

C

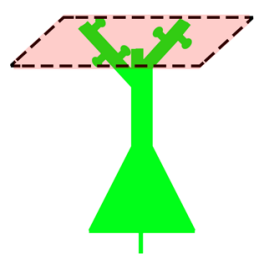

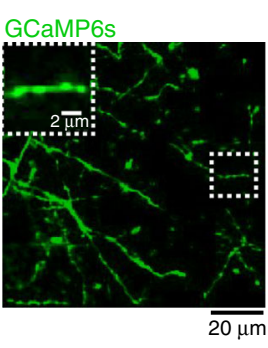

b

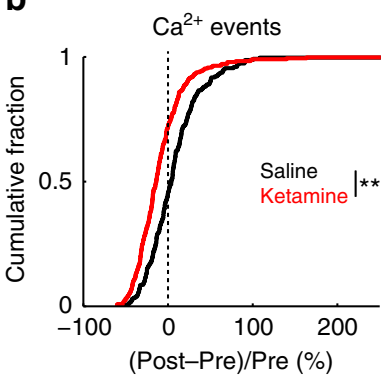

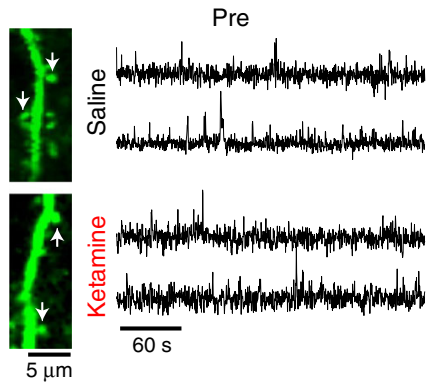
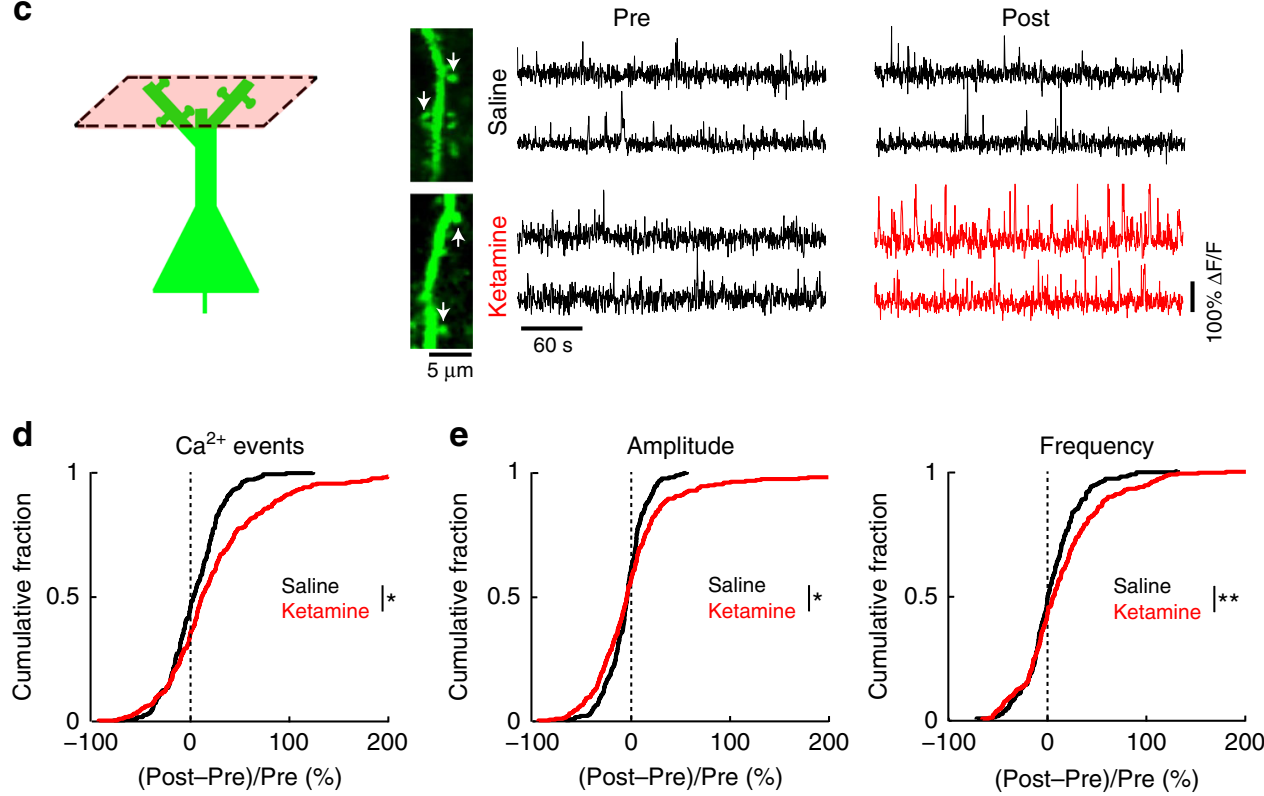

Fig. 2 Ketamine induces opposing effects on dendrite-targeting SST axons and apical dendrites of pyramidal neurons. a Schematic of imaging location, and an in vivo two-photon image of GCaMP6s-expressing SST axons in superficial layers of Cg1/M2 of SST-IRES-Cre animals. Inset, magnified view of a SST axonal segment. $\mathbf{b}$ The normalized difference in the rate of spontaneous calcium events for SST axons in superficial layers. Normalized difference was calculated as post-injection minus pre-injection values normalized by the pre-injection value (ketamine (10 mg/kg): $-9 \pm 3 \%$, saline: $12 \pm 7 \%$, mean \pm s.e. m.; $P=0.002$, two-sample t-test). For ketamine, $n=269$ boutons from 5 animals. For saline, $n=214$ boutons from 5 animals. $\mathbf{c}$ Left, schematic of imaging location. Right, each row shows time-lapse fluorescence transients from the same dendritic spine in the pre-injection (left) and post-injection (right) periods. Two example spines were plotted for saline injection (black) and two other examples were plotted for ketamine (10 mg/kg) injection (preinjection: black; post-injection: red). Locations of the spines in the in vivo two-photon images are indicated by white arrows. $\mathbf{d}$ Same as (b) but for dendritic spines in superficial layers of Cg1/M2 (ketamine $(10 \mathrm{mg} / \mathrm{kg}$ ): $43.42 \pm 0.01 \%$, saline: $4.34 \pm 0.01 \%$, mean \pm s.e.m.; $P=0.02$, two-sample $t$-test). For ketamine, $n=280$ dendritic spines from 5 animals. For saline, $n=231$ dendritic spines from 5 animals. e The normalized difference in amplitude (ketamine $(10 \mathrm{mg} / \mathrm{kg}): 5 \pm 4 \%$, saline: $-4 \pm 1 \%$, mean \pm s.e.m.; $P=0.03$, two-sample $t$-test), and frequency of binned calcium events (ketamine $(10 \mathrm{mg} / \mathrm{kg}): 16 \pm 4 \%$; saline: $4 \pm 2 \%$; $P=0.008$, two-sample $t$-test). ${ }^{\star} P<0.05$; ${ }^{\star \star} P<0.01$; ${ }^{\star \star \star} P<0.001$; n.s., not significant.

pre-pulse intensity, $\mathrm{F}(2,12)=27.5, P=4 \times 10^{-6}$, and drug, $\mathrm{F}$ $(1,24)=2.0, P=2 \times 10^{-4}$, two-way within-subjects ANOVA); however, it had no effect in mice with bilateral GluN2B-SST KD (main effect of pre-pulse intensity, $\mathrm{F}(2,24)=6.3, P=1.7 \times 10^{-4}$, but non-significant drug effect, $\mathrm{F}(1,12)=4.0, P=0.93$; Fig. $6 \mathrm{c}$ ). Bilateral GluN2B-SST $\mathrm{KD}$ in $\mathrm{Cg} 1 / \mathrm{M} 2$ did not relieve all ketamine-induced abnormalities, as hyperlocomotion persisted $(10 \mathrm{mg} / \mathrm{kg}$; Fig. $6 \mathrm{~d})$. These results suggest that the effects of ketamine on some, but not all, behaviors rely on perturbing dendritic inhibition.

Prefrontal GluN2B-SST KD reduced the activity of SST interneurons (Fig. 5c) and increased the rate of spontaneous synaptic calcium transients in pyramidal neurons (Fig. 5e). Although the manipulation rendered ketamine ineffective in trace fear learning and pre-pulse inhibition (Fig. 6b, c), GluN2B-SST $\mathrm{KD}$ alone had no detectable effect on these behaviors. Why does prefrontal GluN2B-SST KD reproduce ketamine's effects on neural dynamics but not the behavioral outcomes? We hypothesized that the discrepancy may arise due to compensatory mechanisms. More specifically, behavioral testing occurred more than 4 weeks after GluN2B-SST KD, and it is possible that other brain regions become involved to restore behavioral functions ${ }^{29}$. To test this hypothesis, we performed experiments in which neural activity was reduced acutely using hM4D(Gi), a designer receptor exclusively activated by designer drug ${ }^{30}$ (DREADD) (Fig. 7a). After Cre-dependent expression of hM4D(Gi) in Cg1/ M2 of SST-IRES-Cre mice, we treated the animals with ligand, clozapine-N-oxide (CNO), or vehicle and repeated the same trace fear conditioning and pre-pulse inhibition experiments along with ketamine or saline treatment. Consistent with the hypothesis, acute reduction of SST interneuron activity via $\mathrm{CNO}+$ $\mathrm{hM} 4 \mathrm{D}(\mathrm{Gi})$ impaired trace fear learning and pre-pulse inhibition in saline-treated animals, while ketamine did not cause any additional effects (Fig. 7b, c). We performed control experiments to show that the effect was not due to hM4D(Gi) expression or $\mathrm{CNO}$ injection injection alone. Thus, unlike the chronic 
$\mathbf{a}_{\text {GCaMP6f }}$
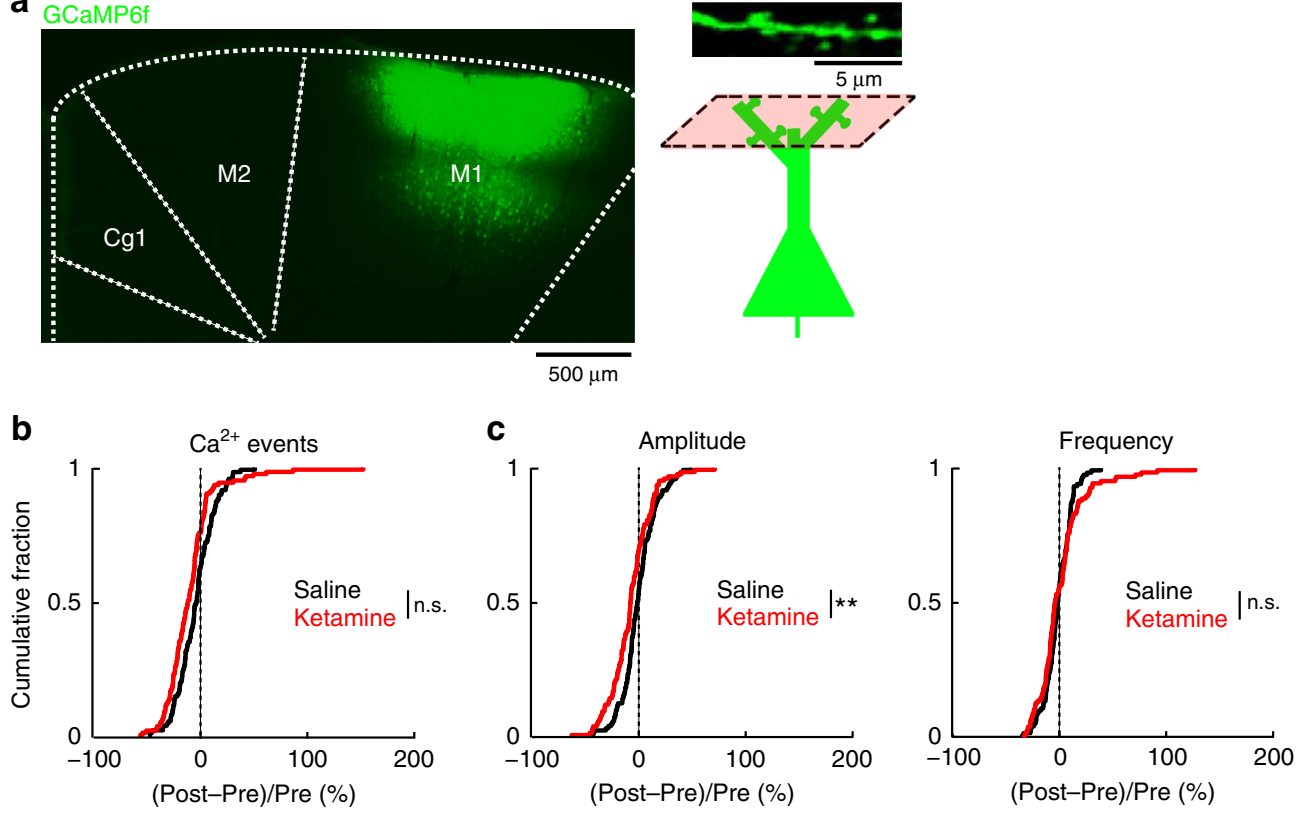

Fig. 3 Effect of ketamine on calcium dynamics for dendritic spines in the primary motor cortex. a Left, coronal histological section, showing the extent of AAV-mediated expression of GCaMP6f in the primary motor cortex, M1. Right, schematic of imaging location. $\mathbf{b}$ The normalized difference in the rate of spontaneous calcium events for apical dendritic spines in M1. Normalized difference was calculated as post-injection minus pre-injection values normalized by the pre-injection value (ketamine $(10 \mathrm{mg} / \mathrm{kg}$ ): $-7 \pm 2 \%$, mean \pm s.e.m.; saline: $-2 \pm 2 \%$ for saline; $P=0.05$, two-sample $t$-test). For ketamine, $n=124$ dendritic spines from 3 animals. For saline, $n=120$ dendritic spines from 3 animals. c The normalized difference in amplitude (ketamine (10 mg/kg): $-7 \pm 2 \%$; saline: $-5 \pm 2 \% ; P=0.004$, two-sample $t$-test) and frequency of binned calcium events $(2 \pm 2 \%$ for ketamine; $-1 \pm 1 \%$ for saline; $P=0.3$, two-sample $t$-test). ${ }^{\star} P<0.05 ;{ }^{\star \star} P<0.01 ;{ }^{\star \star \star} P<0.001 ;$ n.s., not significant.
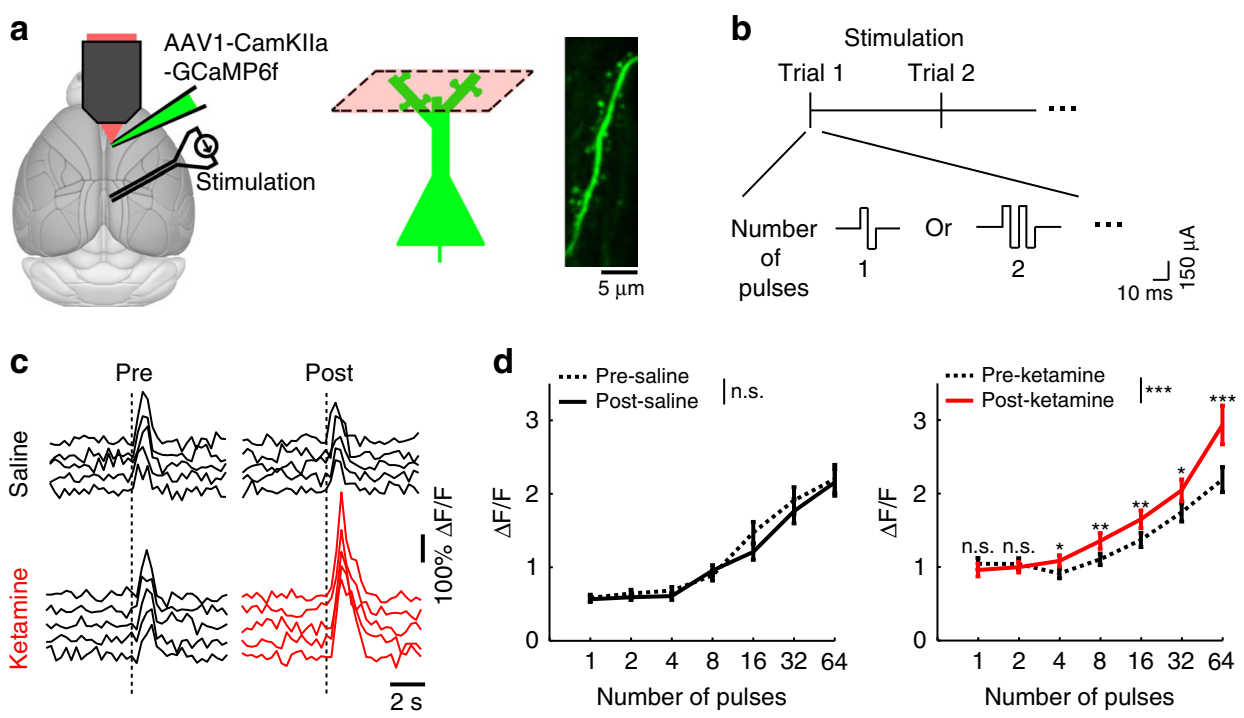

Fig. 4 Ketamine increases synaptically evoked calcium responses in dendritic spines. a Schematic of experimental setup and imaging location. Right, an in vivo two-photon image of GCaMP6f-expressing apical dendritic spines in the superficial layer of Cg1/M2. b Protocol for the RSC stimulation. c Fluorescence transients in dendritic spines in Cg1/M2 in response to electrical stimulation of the retrosplenial cortex (RSC). Each trace shows a single 32-pulse-stimulation trial during pre-injection (left) or post-injection (right) period. One example spine was plotted for saline injection (black), and another example spine was plotted for ketamine injection (red). Dashed line, time of stimulation onset. $\mathbf{d}$ Trial-averaged spine calcium responses as a function of the number of stimulation pulses applied in a trial, for pre-injection (dashed line) vs. post-injection period (solid line) and for saline (black line) vs. ketamine (10 mg.kg) injection (red line). A three-way mixed ANOVA was performed with drug (saline, ketamine) as a between-subjects factor, and stimulation levels $(1,2,4,8,16,32,64)$ and epoch (pre-injection, post-injection) as within-subjects factors. A three-way interaction was significant $(F(6,2364)=5.6$, $\left.P=9 \times 10^{-6}\right)$. A subsequent two-way mixed ANOVA was performed separately on the saline and ketamine datasets, which showed a significant two-way interaction between epoch (pre vs. post) and stimulation levels for ketamine $\left(F(6,1146)=6.6, P=7 \times 10^{-7}\right)$, but not for saline $(F(6,1218)=20.0, P=$ 0.06). Post-hoc Tukey-Kramer test on the ketamine dataset indicated significantly elevated spine responses for post-injection vs. pre-injection for stimulation pulse numbers of $4(P=0.01), 8(P=0.007), 16(P=0.008), 32(P=0.02)$, and $64\left(P=9 \times 10^{-5}\right)$. Line, mean \pm s.e.m. For saline, $n=204$ dendritic spines from 4 animals. For ketamine, $n=192$ dendritic spines from 4 animals. Refer to Supplementary Fig. $5 f$ for full distribution. ${ }^{\star} P<0.05$; ${ }^{\star \star} P<0.01 ;{ }^{\star \star \star} P<0.001$; n.s., not significant. Error bars, \pm s.e.m. 
a

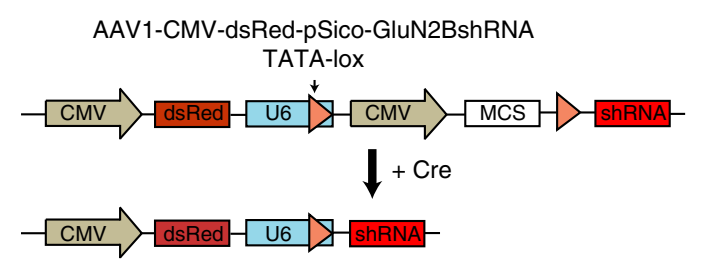

b

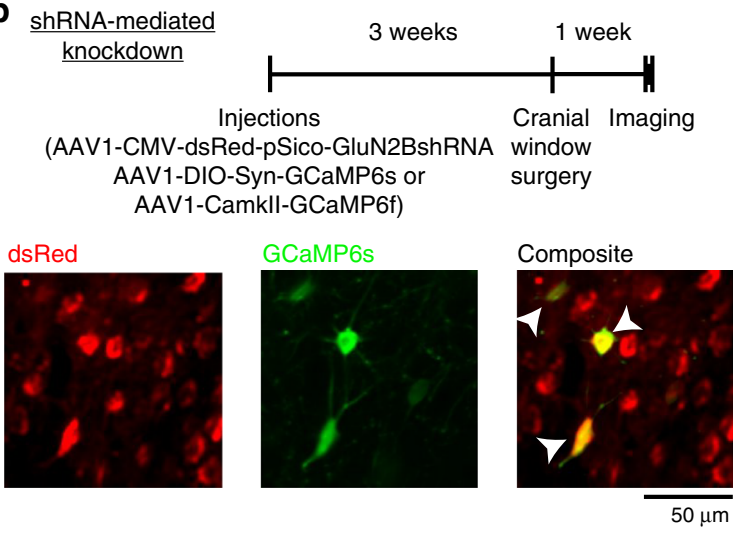

C

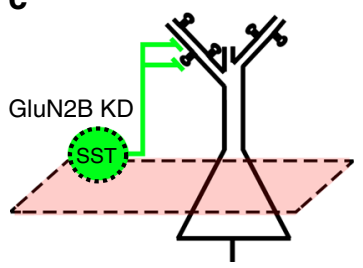

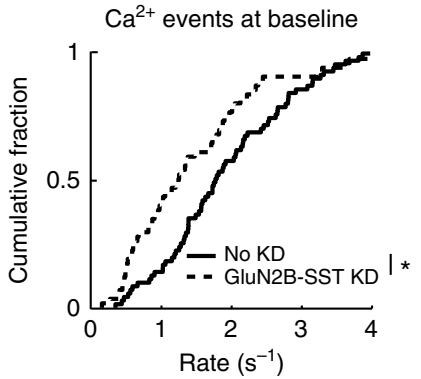

e
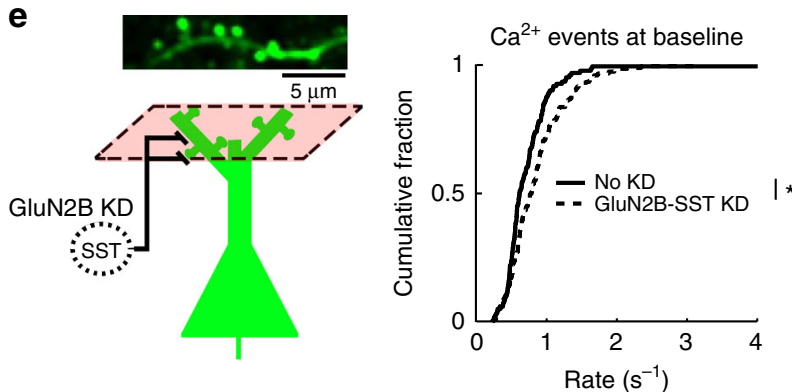

d
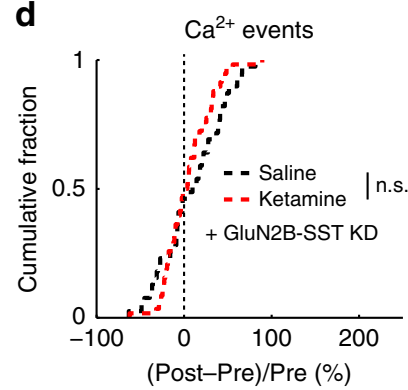

f

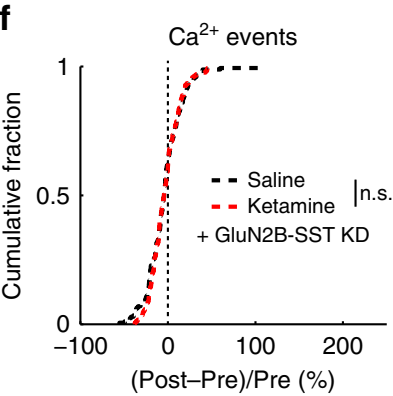

Fig. 5 GluN2B expression in SST interneurons mediates effect of ketamine on dendritic spine calcium. a Schematic of AAV1-CMV-dsRed-pSicoGluN2BshRNA (standard AAV cassettes not drawn) for Cre-dependent expression of shRNA against GluN2B. $\mathbf{b}$ Top, timeline for experiments involving selective knockdown of GluN2B expression in SST interneurons (GluN2B-SST KD). Bottom, in vivo two-photon image of ubiquitous dsRed expression (left), Cre-dependent GCaMP6s expression (middle), and a composite image (right). Arrowheads, cells that expressed both dsRed and GCaMP6s. c The rate of spontaneous calcium events for GCaMP6s-expressing SST interneuron cell bodies with or without GluN2B-SST KD (GluN2B-SST KD: $1.5 \pm 0.2 \mathrm{~Hz}$, control SST-IRES-Cre animals with no KD: $1.9 \pm 0.1 \mathrm{~Hz}$, mean \pm s.e.m.; $P=0.04$, two-sample t-test). For GluN2B-SST KD, $n=58$ cells from 3 animals. For control animals, $n=72$ cells from 3 animals. $\mathbf{d}$ The normalized difference in the number of spontaneous calcium events GCaMP6s-expressing SST interneuron cell bodies, for GluN2B-SST KD with ketamine or saline injection. Normalized difference was calculated as post-injection minus pre-injection values normalized by the pre-injection value (ketamine (10 mg/kg) with GluN2B-SST KD: $2 \pm 4 \%$, saline with GluN2B-SST KD: $7 \pm 6 \%$, mean \pm s.e.m.; $P=0.46$, two-sample $t$ test). For ketamine, $n=58$ cells from 3 animals. For saline, $n=39$ cells from 3 animals. e Same as (c) for GCaMP6f-expressing apical dendritic spines (GluN2B-SST KD: $0.85 \pm 0.03 \mathrm{~Hz}$, mean \pm s.e.m., control SST-IRES-Cre animals: $0.73 \pm 0.04 \mathrm{~Hz}$, mean \pm s.e.m.; $P=0.01$, two-sample t-test). For GluN2BSST KD, $n=277$ spines from 4 animals. For control animals, $n=118$ spines from 3 animals. $f$ Same as (d) for GCaMP6f-expressing apical dendritic spines (ketamine $(10 \mathrm{mg} / \mathrm{kg})$ with GluN2B-SST KD: $-4 \pm 1 \%$, saline with GluN2B-SST KD: $-5 \pm 2 \%$, mean \pm s.e.m.; $P=0.8$, two-sample $t$-test). For ketamine, $n=$ 169 spines from 4 animals. For saline, $n=171$ spines from 4 animals. ${ }^{\star} P<0.05$; ${ }^{\star \star} P<0.01$; ${ }^{\star \star \star} P<0.001$; n.s., not significant.

manipulations, an acute downregulation of prefrontal SST interneuron activity fully occludes the behavioral effects of ketamine on trace fear learning and pre-pulse inhibition.

Dendritic disinhibition explains frontal hyperconnectivity. Because we showed elevated synaptic calcium responses to longrange cortico-cortical inputs, an important question is whether the dendritic disinhibition reported here may contribute to abnormal functional connectivity. To measure communication between cortical regions, we recorded local field potentials (LFP) from Cg1/M2 and RSC (Fig. 8a). We characterized the ultra-slow fluctuations in the envelope of the gamma-band activity (Fig. 8a, b), which has been shown to correlate with blood-oxygenationlevel dependent signals ${ }^{31}$. Reducing dendritic inhibition in Cg1/ M2 led to elevated cortico-cortical coupling between Cg1/M2 and RSC, as evidenced by higher interareal correlation and coherence in GluN2B-SST KD compared to control SST-IRES-Cre animals (Fig. 8c). Ketamine (10 mg/kg), which normally also increased cortico-cortical coupling in control animals, had no further effect in mice with GluN2B-SST KD in Cg1/M2 (Fig. 8d). These data demonstrate that the deficient inhibitory control of dendritic compartments contributes to altered connectivity at the network level. 

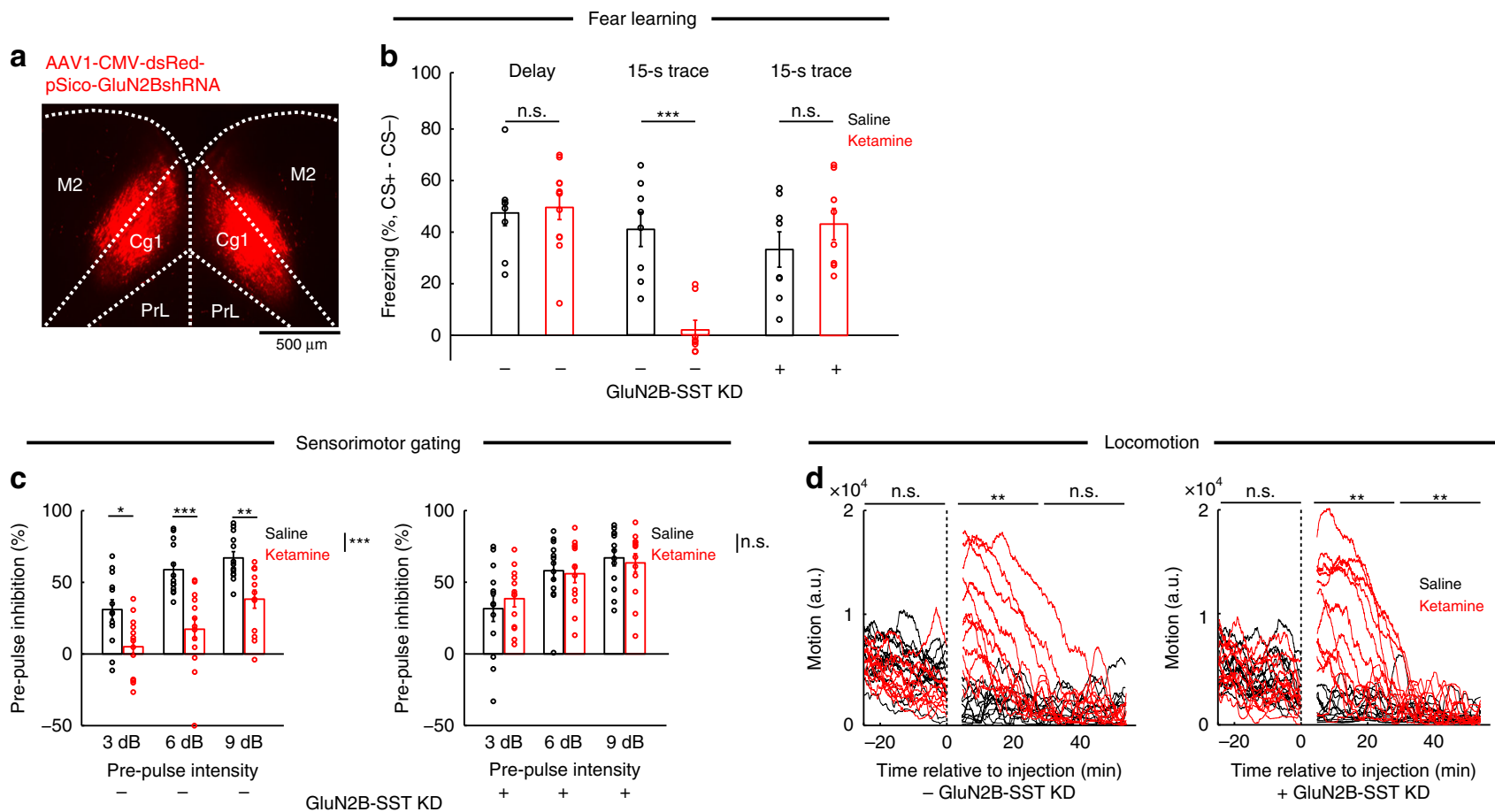

Fig. 6 Chronic downregulation of GluN2B in prefrontal cortical SST interneurons and its behavioral consequences. a Coronal histological section, showing the extent of dsRed expression from bilateral injection of AAV1-CMV-dsRed-pSico-GluN2BshRNA into Cg1/M2 of a SST-IRES-Cre animal. b Fraction of time spent freezing during CS + and trace period (if any), subtracted by that during CS - and trace period (if any), on the testing day. Freezing behavior for control SST-IRES-Cre animals in delay fear conditioning with no trace period (saline: $47 \pm 6 \%$, ketamine $(10 \mathrm{mg} / \mathrm{kg})$ : $50 \pm 5 \%$, mean \pm s.e.m.; $P=0.5$, Wilcoxon rank-sum test; $n=8$ and 12 animals for saline and ketamine, respectively), in trace fear conditioning (saline: $41 \pm 7 \%$, ketamine $(10 \mathrm{mg} / \mathrm{kg}): 2 \pm 4 \%$, mean \pm s.e.m.; $P=6 \times 10^{-6}$, Wilcoxon rank-sum test; $n=8$ animals each for saline and ketamine), and for GluN2B-SST KD animals in trace fear conditioning (saline: $33 \pm 7 \%$, ketamine $(10 \mathrm{mg} / \mathrm{kg}$ ): $43 \pm 6 \%$, mean \pm s.e.m.; $P=0.2$, Wilcoxon rank-sum test; $n=8$ animals each for saline and ketamine). c Pre-pulse inhibition as a measure of sensorimotor gating. For control SST-IRES-Cre animals, a two-way within-subjects ANOVA with prepulse intensity $(3,6$, and $9 \mathrm{~dB})$ and drug (saline, ketamine $(40 \mathrm{mg} / \mathrm{kg})$ ) as within-subjects factors found significant main effects of pre-pulse intensity $\left(F(2,12)=27.5, P=4 \times 10^{-6}\right)$ and drug $\left(F(1,24)=2.0, P=2 \times 10^{-4}\right)$, but a non-significant interaction $(F(2,24) 1.8, P=0.18)$. Post-hoc Tukey-Kramer's tests for saline vs. ketamine were significant at $3 \mathrm{~dB}(40 \pm 7 \%, 5 \pm 6 \%$, mean \pm s.e.m., $P=0.01)$, at $6 \mathrm{~dB}\left(59 \pm 5 \%, 17 \pm 8 \%\right.$, mean \pm s.e.m., $\left.P=9 \times 10^{-4}\right)$, at $9 \mathrm{~dB}(67 \pm 4 \%, 38 \pm 6 \%$, mean \pm s.e.m., $P=0.003) . n=13$ animals each for saline and ketamine. For GluN2B-SST KD animals, two-way within-subjects ANOVA revealed a significant main effect of pre-pulse intensity $\left(F(2,24)=6.3, P=1.7 \times 10^{-4}\right)$, but non-significant drug effect $(F(1,12)=4.0, P=0.93)$ or two-way interaction $(F(2,24)=1.4, P=0.26)$. Saline vs. ketamine yielded no difference at $3 \mathrm{~dB}(32 \pm 9 \%, 39 \pm 6 \%$, mean $\pm \mathrm{s} . e . \mathrm{m}$. $)$, at $6 \mathrm{~dB}(58 \pm 6 \%$, $56 \pm 6 \%$, mean \pm s.e.m.), and at $9 \mathrm{~dB}(67 \pm 6 \%, 63 \pm 6 \%$, mean \pm s.e.m. $) . n=13$ animals each for saline and ketamine. d Open-field locomotor activity. Each trace comes from a single animal. For control SST-IRES-Cre animals, a two-way ANOVA was performed with epoch (pre-injection, 5-30 min post-injection, and 30-60 min post-injection) and drug (saline, ketamine $(10 \mathrm{mg} / \mathrm{kg})$ ) as within-subjects factors. There were significant main effects of epoch $(F(2,22)=$ 21.0, $\left.P=7 \times 10^{-5}\right)$, drug $(F(1,11)=7.1, P=0.02)$, and interaction $\left(F(2,22)=28.5, P=7 \times 10^{-5}\right)$. Post-hoc Tukey-Kramer tests for saline vs. ketamine were significant for 5-30 min post-injection $(P=0.001)$, but non-significant for pre-injection $(P=0.07)$ and 30-60 min post-injection $(P=0.58)$. $n=12$ animals each for saline and ketamine. For GluN2B-SST KD animals, two-way ANOVA revealed significant main effects of epoch $(F(2,22)=14.1, P=0.002)$, drug $(F(1,11)=15.6, P=0.002)$, and interaction $(F(2,22)=13.6, P=0.003)$. Post-hoc Tukey-Kramer tests for saline vs. ketamine were significant for $5-30$ min post-injection epoch $(P=0.003)$ and 30-60 min post-injection $(P=0.001)$, but non-significant for pre-injection $(P=1.0) . n=13$ animals each for saline and ketamine. ${ }^{\star} P<0.05 ;{ }^{\star \star} P<0.01 ;{ }^{\star \star \star} P<0.001$; n.s., not significant. Error bars, \pm s.e.m.

\section{Discussion}

The goal of this study was to delineate the impact of subanesthetic ketamine on the prefrontal cortical circuit. There were two major findings. One, ketamine reduces the activity of dendrite-targeting SST interneurons. Two, as a consequence of the diminished dendritic inhibition, ketamine increases the amplitude of the synaptically evoked calcium transients in the apical dendritic spines of pyramidal neurons. As we will elaborate below, these local-circuit alterations provide insights into the neural mechanisms for the acute psychotomimetic and sustained antidepressant effects of ketamine.

The novelty of our results is to highlight SST interneurons as a key target of systemic NMDAR antagonism. Although in principle the disinhibition hypothesis is general and need not be tied to particular GABAergic neuron subtypes, so far empirical studies of NMDAR hypofunction have focused on fast-spiking cells for technical reasons. For example, recordings of inhibitory postsynaptic currents have provided clear evidence for disinhibition $^{32,33}$, but the method cannot distinguish the cell types that provide the GABAergic inputs, and an electrode placed at the soma tends to favor the detection of perisomatic inputs. In vivo electrophysiology has also supported disinhibition by showing reduced activity for fast-spiking cells ${ }^{11}$, but SST interneurons are likely not examined in this earlier study because their spike waveforms have overlapping features with excitatory neurons and thus cannot be easily isolated ${ }^{16}$. By contrast, using a GABAergic Credriver mouse line, our fluorescence signals would originate predominantly from SST interneurons. The ability to characterize responses from genetically identified SST interneurons distinguishes our results from prior studies of ketamine and neural activity. 
a
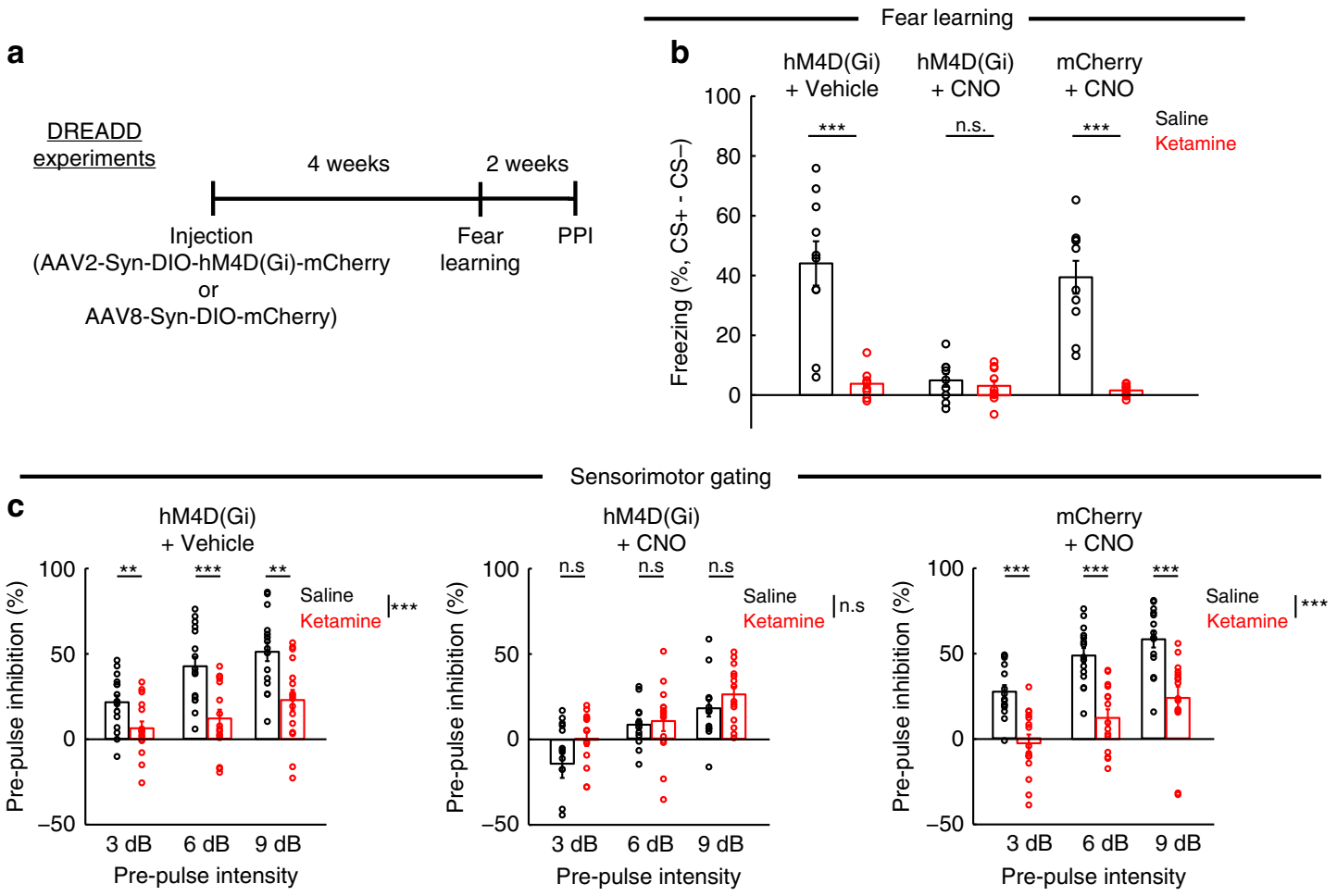

Fig. 7 Acute suppression of SST interneuron activity in Cg1/M2 occludes the behavioral effects of ketamine. a Timeline for experiments involving use of Cre-dependent expression of designer receptors exclusively activated by designer drugs (DREADD) in SST-IRES-Cre animals. b Fraction of time spent freezing during CS + and trace period, subtracted by that during CS - and trace period on the testing day. For hM4D(Gi) + Vehicle condition, ketamine $\left(10 \mathrm{mg} / \mathrm{kg}\right.$ ) treatment caused an impairment in freezing compared to saline (saline: $44 \pm 7 \%$, ketamine: $4 \pm 2 \%$, mean \pm s.e.m.; $P=2 \times 10^{-4}$, Wilcoxon rank-sum test; $n=10$ animals for saline and 9 for ketamine). For hM4D(Gi) + CNO condition, there was impairment in freezing in saline-treated animals with no further difference relative to ketamine-treated $(10 \mathrm{mg} / \mathrm{kg}$ ) animals (saline: $5 \pm 2 \%$, ketamine: $3 \pm 2 \%$, mean \pm s.e.m.; $P=0.78$, Wilcoxon rank-sum test; $n=9$ animals for saline and 10 for ketamine). There was no effect of CNO on ketamine-induced $(10 \mathrm{mg} / \mathrm{kg})$ impairment as tested in the mCherry + CNO condition (saline: $39 \pm 5 \%$, ketamine: $1.5 \pm 1 \%$, mean \pm s.e.m.; $P=2 \times 10^{-4}$, Wilcoxon rank-sum test; $n=10$ animals each for saline and ketamine). c Pre-pulse inhibition as a measure of sensorimotor gating. For $\mathrm{hM} 4 \mathrm{D}(\mathrm{Gi})+$ Vehicle condition, a two-way within-subjects ANOVA with pre-pulse intensity $(3,6$, and $9 \mathrm{~dB})$ and drug (saline, ketamine $(40 \mathrm{mg} / \mathrm{kg}))$ as within-subjects factors found significant main effects of pre-pulse intensity $(F(2,30)=30.6$, $\left.P=6 \times 10^{-7}\right)$ and drug $\left(F(1,15)=21.9, P=3 \times 10^{-4}\right)$, and a significant interaction $(F(2,30)=4.0, P=0.03)$. Post-hoc Tukey-Kramer's tests for saline vs. ketamine were significant at $3 \mathrm{~dB}(22 \pm 4 \%, 6 \pm 4 \%$, mean \pm s.e.m., $P=0.01)$, at $6 \mathrm{~dB}\left(42 \pm 6 \%, 12 \pm 5 \%\right.$, mean \pm s.e.m., $\left.P=1 \times 10^{-4}\right)$, at $9 \mathrm{~dB}(51 \pm 5 \%$, $23 \pm 6 \%$, mean \pm s.e.m., $P=0.002) . n=16$ animals each for saline and ketamine. For hM4D(Gi) + CNO condition, a two-way within-subjects ANOVA found a significant main effect of pre-pulse intensity $\left(F(2,26)=11.9, P=2 \times 10^{-4}\right)$ but a non-significant main effect of drug $(F(1,13)=4.1 P=0.06)$ and interaction $(F(2,26)=1.2, P=0.3)$. Post-hoc Tukey-Kramer's tests for saline vs. ketamine were not significant at $3 \mathrm{~dB}(-14 \pm 8 \%, 1 \pm 4 \%$, mean \pm s.e.m., $P=0.06)$, at $6 \mathrm{~dB}(8 \pm 3 \%, 10 \pm 6 \%$, mean \pm s.e.m., $P=0.71)$, at $9 \mathrm{~dB}(17 \pm 5 \%, 26 \pm 4 \%$, mean \pm s.e.m., $P=0.16) . n=14$ animals each for saline and ketamine. For $\mathrm{mCherry}+\mathrm{CNO}$ condition, a two-way within-subjects ANOVA found significant main effects of pre-pulse intensity $(F(2,28)=30.4, P=9 \times$ $\left.10^{-8}\right)$ and drug $\left(F(1,14)=56.0, P=3 \times 10^{-5}\right)$, and a non-significant interaction $(F(2,28)=0.5, P=0.62$. Post-hoc Tukey-Kramer's tests for saline vs. ketamine were significant at $3 \mathrm{~dB}\left(27 \pm 4 \%,-3 \pm 5 \%\right.$, mean \pm s.e.m., $\left.P=5 \times 10^{-4}\right)$, at $6 \mathrm{~dB}\left(49 \pm 4 \%, 12 \pm 5 \%\right.$, mean \pm s.e.m., $\left.P=1 \times 10^{-5}\right)$, at $9 \mathrm{~dB}(58 \pm$ $5 \%, 24 \pm 7 \%$, mean \pm s.e.m., $P=1 \times 10^{-5}$ ). $n=16$ animals each for saline and ketamine. ${ }^{\star} P<0.05 ;{ }^{\star \star} P<0.01 ;{ }^{\star \star \star} P<0.001$; n.s., not significant.

Perisomatic and dendritic disinhibition may represent separate, parallel ways by which ketamine acts on the prefrontal cortex. This is probable because when we manipulated dendritic inhibition, the behavioral and electrophysiological alterations are largely orthogonal to those previously reported for NMDAR manipulations in fast-spiking cells. More specifically, when the obligatory GluN1 subunit is deleted from fast-spiking cells, behavioral deficits were reported for delay fear conditioning, but not for pre-pulse inhibition ${ }^{34}$. This is opposite to what we saw. In another study with postnatal GluN1 removal in many fast-spiking cells, although there was a deficit in pre-pulse inhibition similar to our animals, the NMDAR antagonist-induced locomotion was reduced $^{35}$, unlike our manipulation of SST interneurons which had no effect on the ketamine-induced hyperlocomotion. Finally, enhanced baseline gamma-band oscillations were observed following NMDAR deletion in fast-spiking cells ${ }^{34,36}$ and may even be produced by removing NMDARs in pyramidal neurons ${ }^{37}$, whereas we show that dendritic inhibition was involved in controlling the envelope of oscillatory signals on a much slower timescale. This comparison comes with the caveat that we are interpreting behavioral outcomes from studies involving NMDAR manipulations to different extent and at different developmental time points. Notwithstanding the caveat, it seems possible that ketamine's suppressive effects on perisomatic and dendritic inhibition may be responsible for non-overlapping sets of behavioral and electrophysiological phenotypes.

We show that the ketamine-induced loss of dendritic inhibition leads to heightened synaptic calcium transients in the dendritic spines of pyramidal neurons. This is consistent with what we know about SST interneurons: they can regulate dendritic excitability and control the amount of calcium influx in dendritic spines in prefrontal cortical brain slices ${ }^{38,39}$. In an awake mouse, 

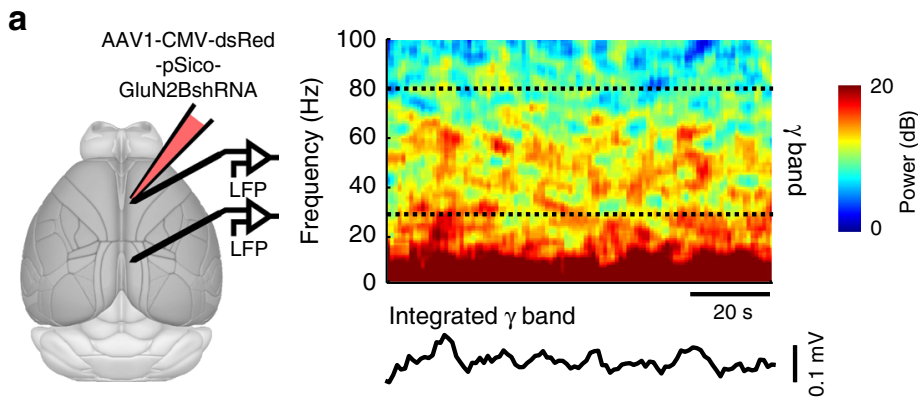

b

C $\mathrm{Cg} 1 / \mathrm{M} 2-\mathrm{RSC}$ integrated $\gamma$ band at baseline

d
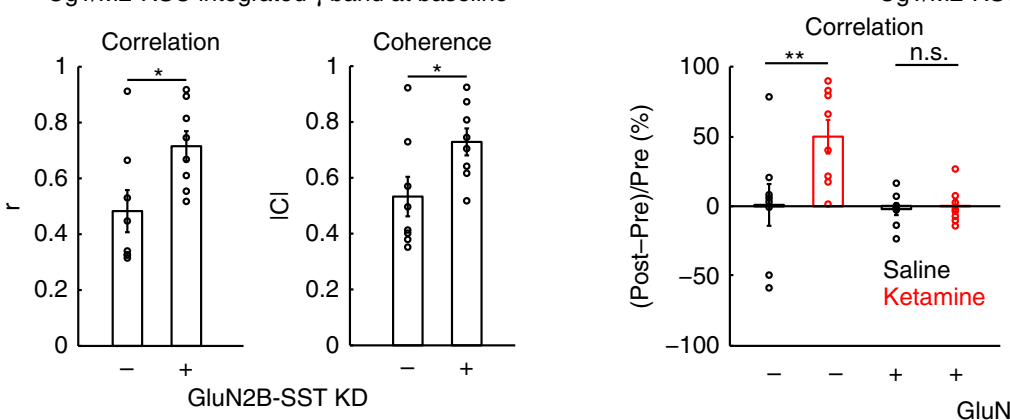

Cg1/M2-RSC integrated $\gamma$ band
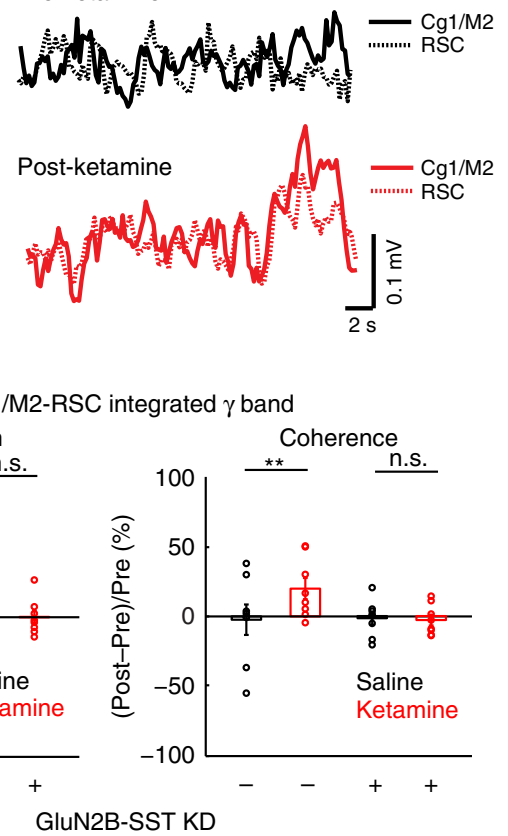

Fig. 8 Elevated prefrontal cortical functional connectivity depends on dendritic inhibition. a Left, schematic of experimental setup. Right, spectrogram of an example local field potential (LFP) recording in Cg1/M2 of a SST-IRES-Cre animal. Dashed lines indicate the upper and lower bounds of the frequency range used to calculate the integrated gamma band signal, which is plotted below the spectrogram. $\mathbf{b}$ Integrated gamma band signals from LFPs recorded simultaneously from Cg1/M2 (solid line) and RSC (dashed line) of a SST-IRES-Cre animal. Example signals were plotted before (black) and after (red) ketamine $(10 \mathrm{mg} / \mathrm{kg}$ ) injection. c Network-scale effects of prefrontal cortical SST interneuron manipulations (SST-IRES-Cre animals with or without GluN2B-SST KD). Functional connectivity between Cg1/M2 and RSC was quantified by determining the correlation and coherence between integrated gamma band signals of the two brain regions. At baseline prior to injection, control and GluN2B-SST KD animals had significantly different correlation coefficients (controls: $0.48 \pm 0.08$, GluN2B-SST KD: $0.72 \pm 0.05$, mean \pm s.e.m.; $P=0.03$, Wilcoxon rank sum test) and coherence magnitude (controls: $0.53 \pm 0.07$, GluN2B-SST KD: $0.73 \pm 0.05$, mean \pm s.e.m.; $P=0.04$, Wilcoxon rank sum test). $n=8$ animals each for controls and GluN2B-SST KD. d Functional connectivity between $\mathrm{Cg} 1 / \mathrm{M} 2$ and RSC after ketamine $(10 \mathrm{mg} / \mathrm{kg})$ or saline for SST-IRES-Cre animals with or without GluN2B-SST KD. For correlation, a two-way mixed ANOVA was performed with treatment (GluN2B-SST KD, no KD) as the between factor, and drug (saline, ketamine) as the within factor. The interaction effect was significant $(F(1,14)=6.2, P=0.03)$. Post-hoc Tukey-Kramer test showed a significant ketamine-saline difference in the no KD group $(P=0.003)$, but not in the GluN2B-SST KD group $(P=0.9)$. For coherence, the interaction effect was significant $(F(1,14)=7.1$, $P=0.02)$. Post-hoc Tukey-Kramer test showed a significant ketamine-saline difference in the no KD group $(P=0.003)$, but not in the GluN2B-SST KD group $(P=0.8) . n=8$ animals each for controls and GluN2B-SST KD. ${ }^{\star} P<0.05 ;{ }^{\star \star} P<0.01$; ${ }^{\star \star \star} P<0.001$; n.s., not significant.

the inhibitory influence of SST interneurons is expected to be greater, as the cells have high spontaneous firing rates in vivo ${ }^{40}$, setting the stage for the suppression by ketamine. The positive skew in the ketamine-induced increase of synaptic calcium transients (Fig. 2d) suggests that only a subset of dendritic spines were impacted by ketamine. This agrees with anatomical studies, showing that only $\sim 20-50 \%$ of cortical dendritic spines are innervated by SST axonal boutons ${ }^{38,41,42}$. By contrast, for SST interneuron soma (Fig. 1i) and axonal boutons (Fig. 2b), ketamine appears to induce a subtractive shift of the entire activity distribution, which is consistent with most SST neurons having NMDAR-mediated currents ${ }^{25}$. One intriguing observation is that the synaptic calcium elevations were observed in the medial prefrontal cortex, but not the primary motor cortex. This may be because although the systemic administration is expected to deliver ketamine to all brain regions, the density of cortical SST interneurons is highest in the frontal associations regions ${ }^{43}$. The gradient of cortical interneuron density may explain the heterogeneous, region-specific responses to a global perturbation ${ }^{44}$.

How might the dendritic disinhibition relate to the behavioral effects of subanesthetic ketamine? Acutely, ketamine produces psychotomimetic symptoms, perceptual aberrations, and cognitive impairments. In the mouse, the $\mathrm{Cg} 1 / \mathrm{M} 2$ sub-regions of the medial prefrontal cortex are involved in linking antecedent events to current decisions ${ }^{45}$. Dendritic inhibition is thought to be crucial for the context-dependent gating of inputs ${ }^{46}$. Dysregulation of dendritic inhibition is therefore expected to allow aberrant sensory inputs into the prefrontal cortex, thereby disorganizing the task-specific activity patterns of prefrontal cortical neurons ${ }^{47}$. Experimentally, this matches with our finding of ketamine-induced prefrontal cortical hyperconnectivity. Our mouse experiments were motivated by human neuroimaging studies that have found abnormally high functional connectivity for the prefrontal cortex in humans with ketamine or early-course schizophrenia ${ }^{48,49}$. Crucially, here we have extended to test the potential neural mechanism by showing that the prefrontal cortical hyperconnectivity in the mouse depends on the ability of ketamine to perturb dendritic inhibition.

What about the antidepressant effects of subanesthetic ketamine? One striking consequence of ketamine is the synaptic adaptations in the medial prefrontal cortex that can be detected $24 \mathrm{~h}$ later, including increased dendritic spine density and strengthened synaptic responses ${ }^{50-52}$. It is thought that these positive influences on synapse function might counteract the impaired synaptic transmission observed in rodents after chronic stress $^{53}$ and the reduced synaptic connections in stressed rodents 
and depressed subjects ${ }^{54,55}$. One possibility is that the heightened calcium transients in dendritic spines within the first hour of systemic administration is important for synaptic plasticity. A potential link between the acute calcium elevations and subsequent synaptic strengthening is supported by studies that have shown postsynaptic calcium influx to be required and sufficient to induce activity-dependent synaptic plasticity ${ }^{56,57}$. It agrees with the observation that the antidepressant effect is blunted if ketamine is administered while excitatory transmission is blocked $^{50,58}$, and that manipulations of SST interneurons can tune depressive-like behaviors in rodents ${ }^{21,26,59}$. SST interneurons are also known to connect densely with pyramidal networks $^{60}$ and thereby regulate their correlated firing 61 , which may relate to a very recent observation that ketamine restores coordinated activity in prefrontal ensembles ${ }^{52}$. Therefore, there are reasons to suspect that dendritic disinhibition might be relevant for ketamine's antidepressant effects. Additional studies will be required to more firmly establish the potential relationship.

\section{Methods}

Animals. Adult male mice ( $>10$ weeks of age at the start of experiments) of the C57BL/6J strain (Stock No. 000664, Jackson Laboratory) were used. To selectively image or manipulate SST interneurons, we utilized adult male SST-IRES-Cre ${ }^{62}$ on a mixed C57BL/6 × 129S4/SvJae background (Stock No. 013044, Jackson Laboratory). Mice were group housed (3-5 mice per cage) on a 12:12-h light/dark cycle with free access to food and water. We typically performed experiments during the light cycle. Sample sizes for this study are selected based on numbers generally employed in the field. All experimental procedures were approved by the Institutional Animal Care and Use Committee at Yale University.

Surgery. For all stereotaxic surgical procedures, the mouse was first anesthetized with isoflurane in oxygen (3-4\% during induction, $1-1.5 \%$ for the remainder of the surgery) while placed in a stereotaxic apparatus (David Kopf Instruments). The animal lay on top of a water-circulating pad (Gaymar Stryker) set at a constant temperature of $38^{\circ} \mathrm{C}$. Eyes were lubricated with ophthalmic ointment. Carprofen $(5 \mathrm{mg} / \mathrm{kg}$, s.c.; 024751, Henry Schein Animal Health) and dexamethasone (3 mg/kg, i.m.; 002459, Henry Schein Animal Health) was used pre-operatively. The skin above the skull was then sterilized by swabs of ethanol and betadine before an incision was made to expose the skull. We made a craniotomy (small or large depending on the experiment as detailed below) using a dental drill. Immediately after surgery and on each day for the following 3 days, carprofen $(5 \mathrm{mg} / \mathrm{kg}$, s.c.) was given to the animal. All animals had at least 1 week to recover before starting experiments unless stated otherwise.

For imaging, we made a small skin incision and craniotomy $(\sim 0.5 \mathrm{~mm}$ diameter) in the right hemisphere centered at $1.5 \mathrm{~mm}$ anterior of bregma (anteriorposterior, $\mathrm{AP}$ ) and $0.3 \mathrm{~mm}$ lateral of midline (medial-lateral, $\mathrm{ML}$ ) to target the medial prefrontal cortex (Cg1 and M2 sub-regions) ${ }^{63}$. A glass pipette (3-000-203G/X, Drummond Scientific) was pulled to a fine tip (P-97 Flaming/Brown Micropipette Puller, Sutter Instrument), front-filled with the relevant viruses (see below) and then slowly lowered into the brain using the stereotaxic apparatus at 4 sites corresponding to vertices of a $0.2-\mathrm{mm}$ wide square centered at the above coordinates. At depth of 0.3 to $0.5 \mathrm{~mm}$ below the dura, we ejected the virus using a microinjector (Nanoject II, Drummond Scientific). To target the retrosplenial cortex (RSC) and primary motor cortex (M1), we used the same approach centering at $\mathrm{AP}=-1.4 \mathrm{~mm} / \mathrm{ML}=0.3 \mathrm{~mm}$, and $\mathrm{AP}=1.8 \mathrm{~mm} / \mathrm{ML}=1.6 \mathrm{~mm}$, respectively. We injected 9.2 or $18.2 \mathrm{nl}$ per injection pulse, with $\sim 30 \mathrm{~s}$ in between each injection pulse. To reduce backflow of the virus, we waited at least $5 \mathrm{~min}$ after finishing injection at one site before retracting the pipette to move on to the next site. For experiments that required more than one virus, we injected each virus sequentially. The brain was kept moist with artificial cerebrospinal fluid (ACSF, in mM: $5 \mathrm{KCl}, 5$ HEPES, $135 \mathrm{NaCl}, 1 \mathrm{MgCl} 2,1.8 \mathrm{CaCl} 2$; $\mathrm{pH}$ 7.3). After completing all injections, the craniotomy was covered with silicone elastomer $(0318$, Smooth-On, Inc.), and the skin sutured (Henry Schein). After $\sim 3$ weeks, the animal underwent a second surgery for cranial window implant. After the initial steps as outlined above, an incision was made to remove all of the skin above the skull, which was cleaned to remove connective tissues. We made a 3-mm diameter craniotomy around the previously targeted location. A glass window was put together by gluing two 3-mmdiameter, \#1-thickness circular glass (640720, Warner Instruments), using UVsensitive optical adhesive (NOA61, Norland Products). The glass window was then placed on the exposed brain with slight downward pressure while applying highviscosity adhesive (Loctite 454) around the perimeter of the glass window. After the adhesive cured, a custom-made stainless steel headplate (eMachineShop.com) was affixed to the skull using quick adhesive cement (C\&B Metabond, Parkell).

For behavior, we followed the surgical procedures for imaging, with the following exceptions. There was no second surgery for cranial window and head plate implant. All injections were bilateral injections, in which we used one of the coordinates above, plus the mirrored coordinates for the other hemisphere. We injected at all the locations in one hemisphere first, before front-filling more virus (if necessary), and moving onto the other hemisphere. We waited at least 4 weeks before behavioral testing.

For local field potential recordings, we fabricated bundles of stainless steel wire electrodes (2-3 wires per bundle) (790500, A-M Systems). The diameter of an electrode was $114.3 \mu \mathrm{m}$ and $50.8 \mu \mathrm{m}$ with and without coating, respectively. There was $\sim 350 \mu \mathrm{m}$ of exposed tip for each electrode which was separated by $\sim 250 \mu \mathrm{m}$ from other electrodes in the bundle. Impedance was typically less than $100 \mathrm{k} \Omega$ when tested at $1 \mathrm{kHz}$. Following the procedures above for surgery and small craniotomy, a bundle of electrodes was each lowered into the right Cg1/M2 and right RSC, centered at the stereotaxic coordinates as described above, to a depth of $0.5 \mathrm{~mm}$ below the dura. We used a miniature stainless steel screw (\#0000-160) placed over the right cerebellum as the reference electrode. All electrodes were then soldered to a miniature connector (A79002-001 or A79000-001, Omnetics). Care was taken to avoid shorted connections. All pieces of the implant along with a stainless steel headplate were affixed to the skull using cyanoacrylate (Loctite 454) and quick adhesive cement (C\&B Metabond, Parkell). For experiments combining viral injections and electrophysiology, we first injected the virus, and then waited 3 weeks before proceeding with a second surgery for electrode implant.

For electrical stimulation, we first injected viruses using the procedures above. Three weeks later, we performed a second surgery to implant stimulating electrodes. A bundle of stainless steel wires (3-4 wires per bundle) (790500, A-M Systems) was inserted into RSC. The wire implant was soldered to a miniature connector (A79002-001 or A79000-001, Omnetics). A glass-covered cranial window was made over $\operatorname{Cg} 1 / \mathrm{M} 2$ using procedures described above. All pieces of the microstimulation implant and a stainless steel headplate were affixed to the skull using cyanoacrylate (Loctite 454) and quick adhesive cement (C\&B Metabond, Parkell).

Histology. Mice were transcardially perfused with paraformaldehyde solution $(4 \%(\mathrm{v} / \mathrm{v})$ in phosphate-buffered saline). The brains stayed in the fixative for at least $48 \mathrm{~h}$, and then were sectioned coronally with a vibratome and mounted on slides with coverslips. We imaged the sections with an upright fluorescence microscope (Zeiss Axio Imager M2).

Viruses. To image calcium transients in pyramidal neurons and their dendrites, we injected AAV1-CamKII-GCaMP6f-WPRE-SV40 (Penn Vector Core) diluted to a titer of $2 \times 10^{12}$ genome copies (GC) per milliliter. A total of $73.6 \mathrm{nl}$ of virus was injected for each experiment. For interneurons, we injected AAV1-Syn-DIOGCaMP6s-WPRE-SV40 (Penn Vector Core; $1 \times 10^{12} \mathrm{GC} / \mathrm{ml}$ titer, $147.2 \mathrm{nl}$ ) in SST IRES-Cre animals. To downregulate GluN2B expression in a cell-type-specific manner, we designed a shRNA sequence targeting mouse GluN2B ( $5^{\prime}$ TGTACCA ACAGGTCTCACCTTAAACTTCAAGAGAGTTTAAGGTGAGACCTGTTGGT ACTTTTTTC $3^{\prime}$ ), ligated the GluN2BshRNA sequence into a modified pSico plasmid backbone ${ }^{21}$, and packaged the plasmid into an AAV to create AAV1CMV-dsRed-pSico-GFP-GluN2BshRNA. For behavioral experiments, a total of $966 \mathrm{nl}$ was injected in Cg1/M2 of each hemisphere, as well as at additional sites around $\mathrm{Cg} 1 / \mathrm{M} 2$ in the anterior-posterior axis. For imaging experiments, the GluN2BshRNA sequence was ligated into another pSico backbone with no GFP (Vector Biolabs; AAV1-CMV-dsRed-pSico-GluN2BshRNA, $2 \times 10^{13} \mathrm{GC} / \mathrm{ml}$ titer) The reason for removing the GFP is to avoid interference with GCaMP6f fluorescence. A total of $552 \mathrm{~nL}$ was injected in $\mathrm{Cg} 1 / \mathrm{M} 2$, and then followed by injection of AAV1-CamKII-GCaMP6f-WPRE-SV40. For electrophysiology experiments, a total of $552 \mathrm{nl}$ of AAV1-CMV-dsRed-pSico-GluN2BshRNA was injected in Cg1/M2. To acutely downregulate SST interneuron activity, we injected either AAV2-Syn-DIO-hM4D(Gi)-mCherry (Penn Vector Core, $1 \times 10^{13} \mathrm{GC} / \mathrm{ml}$ titer, $552 \mathrm{nl})$ for the hM4D(Gi) group or AAV8-Syn-DIO-mCherry (Penn Vector Core $1 \times 10^{13} \mathrm{GC} / \mathrm{ml}$ titer, $552 \mathrm{nl}$ ) for the control group in SST-IRES-Cre animals.

Cell culture validation. We used primary neuronal cell cultures to validate the viral-mediated manipulation of NMDAR expression. Briefly, cortical neurons at E18 from pregnant female rats were dissected, dissociated, and plated in wells $\left(\sim 1\right.$ million cells/well) and cultured in standard media ${ }^{64}$. We added viruses at DIV5 stage. For GluN2BshRNA validation, we used the viruses AAV1-CMV-Cre GFP $\left(1 \times 10^{13} \mathrm{GC} / \mathrm{ml}\right.$ titer, $\left.400 \mathrm{nl}\right)$ for expression of Cre and AAV1-CMV-dsRedpSico-GluN2BshRNA $\left(2 \times 10^{13} \mathrm{GC} / \mathrm{ml}\right.$ titer, $\left.400 \mathrm{nl}\right)$ for expression of GluN2Bsh RNA. Using these two viruses, we made four well conditions: GluN2BshRNA, no Cre; GluN2BshRNA, Cre; no GluN2BshRNA, Cre; no GluN2BshRNA, no Cre (no virus added) (Supplementary Fig. 6). We then performed Western blots with antibodies for GluN2B (\#4207S, Cell Signaling, 1:1000, rabbit polyclonal antibody) and beta-actin (\#4967S, Cell Signaling, 1:1000, rabbit polyclonal antibody). Bands were quantified using Bio-Rad Image Lab software and raw band intensity values were normalized to beta-actin levels.

Drugs. We used saline (0.9\% Sodium chloride, Hospira). Ketamine working solution ( $1 \mathrm{mg} / \mathrm{ml}$ in saline) was prepared from stock solution (McKesson), approximately every 30 days. All ketamine and saline injections were subcutaneous (s.c.). All imaging, locomotor activity and fear conditioning experiments used 
$10 \mathrm{mg} / \mathrm{kg}$. Only the PPI experiments used a higher dose of $40 \mathrm{mg} / \mathrm{kg}$. Initial testing at $10 \mathrm{mg} / \mathrm{kg}$ in control mice did not yield reliable effects on PPI performance. We therefore used a higher dosage, following a prior study ${ }^{65}$. Clozapine N-oxide (CNO) (Sigma Millipore) working solution $(1 \mathrm{mg} / \mathrm{ml}$ ) was prepared fresh daily before each experiment from a stock solution dissolved in DMSO $(10 \mathrm{mg} / \mathrm{ml})$ stored at $4{ }^{\circ} \mathrm{C}$. Vehicle (1:10 DMSO in saline) was used in control injections. All $\mathrm{CNO}$ and vehicle injections were intraperitoneal (i.p.).

Two-photon calcium imaging. We used a laser-scanning two-photon microscope (Movable Objective Microscope, Sutter Instrument) controlled by the ScanImage software $^{66}$. A tunable Ti:Sapphire femotosecond laser (Chameleon Ultra II, Coherent) was the excitation source which was focused onto the brain using a water immersion objective (XLUMPLFLN, $\times 20 / 0.95$ N.A., Olympus). The timeaveraged laser power was typically less than $100 \mathrm{~mW}$ during experiments. Filters had the following center excitation (ex) and emission (em) wavelengths (Semrock or Chroma): GCaMP6s/f, $920 \mathrm{~nm}(\mathrm{ex})$ and $525 \mathrm{~nm}(\mathrm{em})$; dsRed, $1040 \mathrm{~nm}(\mathrm{ex})$ and $605 \mathrm{~nm}(\mathrm{em})$. Emitted photons were collected by GaAsP photomultiplier tubes. We acquired images that were $256 \times 256$ pixels at 0.68 or $0.54 \mu \mathrm{m}$ per pixel resolution for dendritic spines and axonal boutons or $1.35 \mu \mathrm{m}$ per pixel resolution for cell bodies. Frame rate was $3.62 \mathrm{~Hz}$ using bidirectional scanning on a set of galvanometer-based scanners. A software program (Presentation, NeuroBehavioral Systems) sent TTL pulse via a data acquisition device (USB-201, Measurement Computing) to the two-photon microscope at 30-s intervals, as well as to trigger all the other equipment. The Presentation software would log timestamps so that data from imaging and other equipment (e.g., microstimulation and body motion) can be synchronized in later analyses. Mice were habituated to head fixation under the microscope for 3-5 days of increasing durations before the commencement of any data collection. While head fixed, the mouse would sit in an acrylic tube, which permits postural adjustments but limits gross body movements. Subcutaneous injections were given through a drilled hole in the tube so as to allow the mouse to remain head fixed. To examine the acute effects of ketamine, we would image a field of view for 10-15 min to obtain 'pre-injection' data. The imaging was then interrupted for ketamine $(10 \mathrm{mg} / \mathrm{kg})$ or saline $(10 \mathrm{ml} / \mathrm{kg})$ injection (s.c.). At $30 \mathrm{~min}$ after the injection, we would verify that the same field of view was still in focus, and then obtain imaging data for another 10-15 min. Imaging $30 \mathrm{~min}$ after the injection ensured that we bypass the transient hyperlocomotion effects of ketamine (Fig. 1c, d). In a subset of animals, we imaged continuously for $60 \mathrm{~min}$ after injection, but analyzed only the data after the 30 -min post-injection mark. Each subject was imaged for ketamine and saline injections, with the order counterbalanced across subjects. We imaged at $0-200 \mu \mathrm{m}$ below dura for dendritic spines, and $200-500 \mu \mathrm{m}$ below dura for cell bodies. We targeted the medial portion of $\mathrm{Cg} 1 / \mathrm{M} 2$, within $0-500 \mu \mathrm{m}$ of the midline as visualized by the dark band of the midsagittal sinus. For imaging experiments with cell-type-specific knockdown experiments, during live imaging, we searched for and imaged fields of view with many cells doublelabeled with dsRed and GCaMP6s.

Analysis of calcium imaging data. We first concatenated all of the tiff image files from the same experiment and corrected for lateral motion using either TurboReg implemented as a plugin in ImageJ or NoRMCorre ${ }^{67}$ in MATLAB. A custom graphical user interface (GUI) in MATLAB was used for the manual selection of region of interests (ROI). The rest of the analysis depended on experiment type as described below.

For dendritic spines, we manually scrolled through the imaging frames to look for ROIs that likely represented dendritic spines and dendritic shaft-neurite segments with multiple protrusions that display correlated patterns of fluorescence transients. The dendritic shaft ROIs were selected to be within $20 \mu \mathrm{m}$ of the corresponding dendritic spine ROIs. We averaged values of pixels within a ROI to generate $F_{\mathrm{ROI}}(t)$. For each ROI, we quantified the contribution of neuropil to the fluorescence signal. Specifically, we would calculate a radius, $r$, treating the ROI area as the area of a circle, and then creating an annulus-shaped neuropil ROI with inner and outer radii of $2 r$ and $3 r$, respectively. We excluded pixels that belonged to the ROIs of other spines and shaft. To also exclude pixels that may belong to unselected dendritic structures, we calculated the time-averaged signal for each pixel, and then determined the median for all pixels within the neuropil ROI. We then excluded pixels if their time-averaged signal was higher than the median. We finally averaged across the non-excluded pixels in the neuropil ROI to generate $F_{\text {neuropil }}(t)$. To subtract the neuropil signal, we used the formula:

$$
F(t)=F_{\mathrm{ROI}}(t)-c F_{\text {neuropil }}(t)
$$

where $c=0.4$ is the neuropil correction factor. The fractional change in fluorescence, $\Delta F / F(t)$, for each spine and shaft was calculated as follows:

$$
\frac{\Delta F}{F}(t)=\frac{F(t)-F_{o}(t)}{F_{o}(t)}
$$

where $F_{0}(t)$ is the 10th percentile of $F(t)$ within a 2-min sliding window. Because we were presently interested in calcium signals due to subthreshold synaptic inputs, we regressed out fluorescence signals from non-local sources ${ }^{17}$. For each spine with $\Delta F / F_{\text {spine }}(t)$, we computed $\Delta F / F_{\text {synaptic }}(t)$ by subtracting out the scaled version of fluorescence transients in the corresponding dendritic shaft, $\Delta F / F_{\text {shaft }}(t)$, using the following equation:

$$
{\frac{\Delta F}{F_{\text {synaptic }}}}(t)=\frac{\Delta F}{F_{\text {spine }}}(t)-\alpha \frac{\Delta F}{F_{\text {shaft }}}(t)
$$

where $\alpha$ was determined by a linear regression forced through the origin of $\Delta F / F_{\text {spine }}(t)$ vs. $\Delta F / F_{\text {shaft }}(t)$.

For axonal boutons, we drew ROIs that are likely single axonal boutonsvaricosities on a relatively thin neurite branch with no protrusions. This manual identification is supported by the known neuroanatomy. For SST interneurons, they send axons but very few dendrites, if any, to layer 1 . For retrosplenial cortical neurons, they send long-range axons to layer 1 of $\mathrm{Cg} 1 / \mathrm{M} 2$. To generate $F_{\mathrm{ROI}}(t)$, values of pixels within an ROI were averaged. For each ROI, we would estimate the contribution of neuropil to the fluorescence signal, by calculating and subtracting $F_{\text {neuropil }}(t)$ to obtain $F_{\text {candidate bouton }}(t)$, using the procedures described above. Some boutons may belong to the same neuron. To limit duplicates, we used a modified a published procedure ${ }^{68}$. Briefly, we first analyzed a subset of imaging experiments to identify pairs of boutons on the same branch, based on distance (within $10 \mu \mathrm{m}$ of each other) and visual confirmation of a connecting axonal segment. From this subset of bouton pairs ( $n=46$ bouton pairs, 2 animals), we used their

$F_{\text {candidate_bouton }}(t)$ values to compute a correlation coefficient for each pair. The 5 th percentile value for all the correlation coefficients in this subset was 0.59 , which was used as the cut-off threshold value. Then, for each axonal imaging experiment, we performed an iterative procedure to select unique boutons. In each iteration, a correlation matrix using $F_{\text {candidate_bouton }}(t)$ between all pairs of candidate boutons in a field of view was computed. The pair of candidate boutons with the highest correlation coefficient was identified. If the value exceeded the threshold of 0.59 , we would select one out of that pair of candidate boutons randomly, and looked for other boutons whose correlation coefficient with the selected bouton exceeded the threshold of 0.59 . The original identified pair and boutons from subsequent search then would form one cluster. Entries in the correlation matrix corresponding to this cluster would be excluded for subsequent iterations. The process continued again to identify another cluster until no pair of candidate boutons in the correlation matrix had value above the threshold of 0.59 . At the end of the iterative procedure, each of the remaining boutons was assigned to be its own cluster. We randomly selected one bouton as the representative for each cluster. The fluorescence signals of these representative boutons, $F_{\text {bouton }}(t)$, were then converted to fractional changes in fluorescence, $\Delta F / F_{\text {bouton }}(t)$, as described above and used for subsequent analyses.

For cell bodies, we selected ROIs that are likely cell bodies-fluorescent objects with a round boundary and a lateral extent of about $10 \mu \mathrm{m}$. Values of pixels within a ROI were averaged to generate $F_{\mathrm{ROI}}(t)$. For each ROI, we would remove the contribution of neuropil to the fluorescence signal, by calculating and subtracting $F_{\text {neuropil }}(t)$ to obtain $F_{\text {soma }}(t)$, and then calculating the fractional changes in fluorescence $\Delta F / F_{\text {somal }}(t)$, using procedures outlined in the previous sections. For experiments with cell-type-specific GluN2B manipulation, we selected only GCaMP6s-expressing cells that were co-labeled with dsRed.

After obtaining $\Delta F / F_{\text {synaptic }}(t), \Delta F / F_{\text {bouton }}(t)$, or $\Delta F / F_{\text {soma }}(t)$ for a ROI, we did the following analysis to detect calcium events. We employed a validated "peeling" algorithm ${ }^{15}$, which used an iterative, template-matching procedure to decompose $\Delta F / F(t)$ into a series of elementary calcium events. We used a template for an elementary calcium event with instantaneous onset, an amplitude of 0.3 , and a decay time constant of $1 \mathrm{~s}$ for a single-exponential function. The algorithm would search for a match to this specified template in $\Delta F / F(t)$. When a match occurred, a calcium event was recorded, and the template subtracted from $\Delta F / F(t)$ (i.e., peeling). The remaining $\Delta F / F$ trace was then searched for another match, in an iterative manner until no further matches were found. The times of recorded calcium events formed the output of the algorithm. The temporal resolution of the output is limited by the imaging frame rate. There can be multiple events associated with the same event time. In the paper describing the peeling algorithm, extensive numerical simulations suggested excellent detection performance, even in worstcase scenarios with low signal-to-noise ratios and low sampling rate ${ }^{15}$. For each imaging session, we calculated calcium event rate by dividing the number of calcium events by the duration of the imaging period. For Figs. $2 \mathrm{e}, 3 \mathrm{c}$, and Supplementary Fig. 1a, c, we characterized the calcium events in terms of amplitude and frequency. We identified calcium events that co-occurred in the same imaging frame (same event time), distinguishing amplitude (mean number of calcium events per frame, for frames with at least one event) from frequency (number of frames with at least one event divided by the duration of the imaging session). We imaged the same compartment (spine, bouton, or cell body) before and after injection, and therefore were able to quantify the change in calcium event rate, amplitude, and frequency for each compartment by calculating the postinjection minus pre-injection values normalized by the pre-injection value.

To determine if our results depend on the event detection procedure, we used an alternative method to quantify calcium transients in dendritic spines (Supplementary Fig. 4). We started from $\Delta F / F_{\text {synaptic }}(t)$. For each ROI and for each condition (i.e., pre-injection or post-injection), we would calculate a threshold corresponding to 3 times the median absolute deviation of $\Delta F / F_{\text {synaptic }}(t)$. We noted imaging frames in which $\Delta F / F_{\text {synaptic }}(t)$ was above the threshold. From this subset of frames, we determined the mean amplitude above threshold $\left(\Delta F / F_{\text {synaptic }}(t)\right.$ minus the threshold), and the fraction of time spent above the threshold (the number of above-threshold frames divided by the number of all frames) (see 
Supplementary Fig. 4a for an illustration). We quantified the changes in these measures-mean amplitude above threshold and fraction of time above threshold -by calculating the post-injection minus pre-injection values normalized by the pre-injection value.

Recording animal movements during two-photon imaging. To assess the mouse's movements during two-photon imaging (Fig. 1c, d), we illuminated the animal with infrared light and recorded videos $(640 \times 480$ pixels, $20 \mathrm{~Hz})$ of the animal's face and body using an infrared camera (See3CAM_12CUNIR, e-con Systems). Although the animal was head-fixed, it could still move parts of its body such as the face, trunk, and limbs. To synchronize the infrared video with imaging, the infrared illuminator was triggered to turn off for $100 \mathrm{~ms}$ by the same TTL pulse used to trigger the two-photon microscope. During analysis, the interval between dark frames therefore indicated a duration of $30 \mathrm{~s}$. We computed motion $m$ in 1-s bins:

$$
m=\frac{\sum_{f=1}^{N-1} \sum_{x=1}^{X} \sum_{y=1}^{Y}\left|p_{x, y}(f+1)-p_{x, y}(f)\right|}{N-1}
$$

where $N$ is the number of video frames in the 1-s bin, $X$ and $Y$ are the width and height of the frame, $p_{x, y}(f+1)$ and $p_{x, y}(f)$ are values of the pixel at coordinates $x$ and $y$ in frames $f+1$ and $f$, respectively.

\section{Simultaneous electrical stimulation and imaging. Imaging in $\mathrm{Cg} 1 / \mathrm{M} 2$ was} performed as described above. For microstimulation, we injected electrical currents into the implanted stimulation electrodes in RSC, using an analog stimulus isolator (Model 2200, A-M Systems). The timing and strength of the stimulation were specified by a script written in a software program (Presentation, NeuroBehavioral Systems) via command voltages out of a multifunctional input/output device (USB6001, National Instruments). During each experiment, to calibrate the stimulation current (which could vary due to depleting battery for the stimulus isolator or high impedance of the implanted electrode), an electronic circuit was placed in-series, allowing us to measure the stimulation current online and tune the electrical current to within $5 \%$ of the desired level. For the stimulation pattern, each pulse had a biphasic waveform, lasted $5 \mathrm{~ms}$ in each phase, and had peak amplitudes of $\pm 150 \mu \mathrm{A}$. We used $1,2,4,8,16,32$, or 64 pulses as the stimulation levels. If more than 1 pulse was used, the pulses were applied one after the other with no interpulse interval. A block consisted of the 7 stimulation levels, presented in random order with a random inter-trial period of 10-14 s. For each experiment, we presented 10 blocks successively before injection of ketamine or saline, and then presented another 10 blocks successively 30-60 min after injection. Prior to the post-injection stimulation, we imaged to confirm that the field of view has not changed. Using procedures described in earlier sections, we determined $\Delta F / F$ in each dendritic spine and axonal bouton. The log files generated by the Presentation software were used to align the timestamps of the imaging data to the stimulation events. For each dendritic spine or axonal bouton, we determined the $\Delta F / F$ measured within $1 \mathrm{~s}$ following the onset of stimulation, and reported the averaged values across the 10 blocks.

Electrophysiology. Animals were head-fixed and placed in a Faraday cage. An interface cable was mated to the connector on the animal's head in order to amplify local field potential (LFP) signals using a differential amplifier (RHD2216, Intan Technology), high-pass filtered at $0.5 \mathrm{~Hz}$ and digitized at $1 \mathrm{kHz}$ (RHD2000, Intan Technology). Before actual electrophysiological recordings, mice were habituated to head-fixation for 3-5 days of increasing durations. We recorded for $30 \mathrm{~min}$ preinjection, and then for $60 \mathrm{~min}$ post-injection. We discarded data from electrodes that were noisy (impedance $>1 \mathrm{G} \Omega$ ). One electrode from each site (Cg1/M2 or RSC) was chosen randomly for analysis. For analysis, we compared the 30-min segment recorded pre-injection to the 30-min segment recorded from 30-60 min post-injection. We focused on the integrated gamma band power, because it was previously found to correlate with hemodynamic signals underlying bloodoxygenation-level-dependent resting-state connectivity ${ }^{31}$. To determine the integrated gamma band power, spectrograms were computed from the local field potential recordings using a sliding window with duration of $2 \mathrm{~s}$ and step size of 0.4 s. At each window, spectral power within the gamma band from 30 to $80 \mathrm{~Hz}$ was summed, and this yielded the integrated gamma band power. Correlation between integrated gamma band power in Cg1/M2 and RSC was calculated based on Pearson correlation coefficient at zero lag. Coherence between integrated gamma band power in Cg1/M2 and RSC was computed using the Chronux ${ }^{69}$ package in MATLAB, and the magnitude of coherence was reported.

Behavior. Fear learning was assessed using a conditioning box utilizing an infrared video camera $(320 \times 240$ pixels, $30 \mathrm{~Hz}$; Med Associates, Inc.). To enable more flexible protocols involving multiple stimuli and repetitions, we modified the box to be controlled by a behavioral control software (Presentation, NeuroBehavioral Systems). Each of the conditioned stimuli (CS) was a 20-s long series of auditory pips ( $500 \mathrm{~ms}$ on, $500 \mathrm{~ms}$ off). The pip was either $2.5 \mathrm{kHz}$ ( $85 \mathrm{~dB}$, calibrated by A-weighting at $15 \mathrm{~cm}$ from speaker) or $11 \mathrm{kHz}(75 \mathrm{~dB})$. For delay conditioning experiments, one CS (CS+) co-terminated with the unconditioned stimulus of footshock (US; $0.65 \mathrm{~mA}, 0.5 \mathrm{~s}$ ), and the other CS (CS-) was not associated with any event. For trace conditioning experiments, the end of CS+ and US was separated by $15 \mathrm{~s}$ of no stimulus. In the acquisition phase, animals were injected with either ketamine $(10 \mathrm{mg} / \mathrm{kg}$, s.c.) or saline $(10 \mathrm{ml} / \mathrm{kg}$, s.c.), returned to the home cage, and then placed in the conditioning box $15 \mathrm{~min}$ later. For DREADD experiments, $\mathrm{CNO}(5 \mathrm{mg} / \mathrm{kg}$, i.p.) or vehicle $(10 \mathrm{mg} / \mathrm{ml}$, i.p.) was injected $30 \mathrm{~min}$ before trace fear conditioning followed by ketamine or saline $15 \mathrm{~min}$ before commencement of conditioning. After 6 min of habituation, conditioning started during which 5 CS - and 5 CS + were presented in a random order with a random inter-trial period of 100-110 s. We counter-balanced across subjects the auditory characteristics of CS+ and CS-, as well as the ketamine and saline injections. In the recall phase, which occurred about $24 \mathrm{~h}$ later, we tested the animals in a different context. Context was altered using different odors, wall inserts, light, and ambient noise. After $6 \mathrm{~min}$ of habituation, testing commenced, during which 4 CS - were presented followed by 4 CS + (with no US) with a random inter-trial period of $100-110 \mathrm{~s}$. Motion was quantified from the infrared video data using automated procedures in the Video Freeze software (Med Associates, Inc.). Briefly, according to the publication related to the software ${ }^{70}$, motion was quantified by calculating the sum of pixel-by-pixel value changes across successive video frames. Time periods when the motion values fell below the software-preset threshold for at least $1 \mathrm{~s}$ were considered freezing periods. For $35 \mathrm{~s}$ after the onset of each CS + or CS- (i.e., the 20-s long CS presentation plus the 15-s long trace period), we determined the fraction of time in which freezing was detected. We reported the differential value, i.e., the fraction of time spent freezing in response to CS+, subtracted by the fraction of time spent freezing in response to CS-, as measured in the recall phase.

To measure sensorimotor gating, we used a commercial system (SR-Lab, San Diego Instruments). An animal would be injected with ketamine ( $40 \mathrm{mg} / \mathrm{kg}$, s.c.) or saline $(10 \mathrm{ml} / \mathrm{kg}$, s.c.) and then immediately placed in a cylinder on a platform with an accelerometer for recording motion, all of which lie inside a soundproof chamber. For DREADD experiments, CNO $(5 \mathrm{mg} / \mathrm{kg}$, i.p.) or vehicle $(10 \mathrm{mg} / \mathrm{ml}$, i.p.) was given $30 \mathrm{~min}$ before injection of ketamine or saline and immediate commencement of PPI. The animal was habituated for $5 \mathrm{~min}$ to background noise ( $66 \mathrm{~dB}$, calibrated by A-weighting at $25 \mathrm{~cm}$ from speaker) and startle stimuli $(120 \mathrm{~dB}$ noise, $50 \mathrm{~ms})$. For testing, there were 5 types of trials: startle, pre-pulse trials during which the onset of a pre-pulse stimulus $(3,6$, or $9 \mathrm{~dB}$ noise above background, $50 \mathrm{~ms}$ ) preceded a startle stimulus by $100 \mathrm{~ms}$, and catch trials with no stimulus. A block consisted of 5 trials, 1 of each type, in a random order with a random inter-trial period of 8-23 s. For each animal, we would test for 10 blocks (i.e., 50 trials in total). The pre-pulse inhibition (PPI) is calculated as follows:

$$
\text { PPI }=\frac{S_{\text {startle }}-S_{\text {prepulse }}}{S_{\text {startle }}}
$$

where $S_{\text {startle }}$ and $S_{\text {prepulse }}$ were the rectified motion signals recorded by the accelerometer during the $50-\mathrm{ms}$ long startle stimulus averaged across trial repeats, for the startle and prepulse trials, respectively.

To assess locomotor activity, we placed animals in a conditioning box equipped with an infrared video camera $(320 \times 240$ pixels, $30 \mathrm{~Hz}$; Med Associates, Inc.). After $\sim 30 \mathrm{~min}$ of habituation, they were injected with either ketamine $(10 \mathrm{mg} / \mathrm{kg}$, s.c.) or saline $(10 \mathrm{ml} / \mathrm{kg}$, s.c.) and returned to the box for another $60 \mathrm{~min}$. Motion was assessed from the infrared video data quantified using automated procedures in the Video Freeze software (Med Associates, Inc.). To plot the results for Fig. 6d, we smoothed the motion signals with a 5 -min long moving window at $1 \mathrm{~s}$ steps, for visualization purposes. For statistical comparisons, we used the timeaveraged motion for each of the epochs: pre-injection, 5-30 min post-injection and $30-60 \mathrm{~min}$ post-injection.

Statistics. Statistical tests as described in figure legends were done in MATLAB. For the imaging experiments, all planned two-group comparisons were done with parametric $t$-tests (two-tailed, $\alpha=0.05$ ). For experiments with multiple parameter and conditions, we employed ANOVA tests, with groupings as indicated in the figure legend, followed by post-hoc tests using Tukey-Kramer for statistically significant main or interaction effects. The sampling distribution of the mean was assumed to be normal, but this was not formally tested. To test the robustness of our results, we also ran all the statistical tests in Figs. 1-3 using the two-sample Kolmogorov-Smirnov (K-S) test. This nonparametric test allows us to compare the two cumulative fraction plots (saline vs. ketamine) directly without any assumption of normality. None of the conclusions of whether to reject the null hypothesis at $a=0.05$ were affected by a change in statistical tests (data not shown). For behavior and electrophysiology experiments, we used non-parametric tests (twotailed, $\alpha=0.05$ ). In the figures, $P$-values were represented as n.s., not significant; $P>0.05 ;{ }^{*} P<0.05 ;{ }^{* *} P<0.01 ;{ }^{* *} P<0.001$. See figure legends for exact $P$-values.

Reporting summary. Further information on research design is available in the Nature Research Reporting Summary linked to this article.

\section{Data availability}

The data that support the findings of this study are available from the corresponding author upon reasonable request. 


\section{Code availability}

MATLAB scripts for the present study are available from the corresponding author upon reasonable request.

Received: 22 April 2019; Accepted: 22 November 2019;

Published online: 07 January 2020

\section{References}

1. Krystal, J. H. et al. Subanesthetic effects of the noncompetitive NMDA antagonist, ketamine, in humans: psychotomimetic, perceptual, cognitive, and neuroendocrine responses. Arch. Gen. Psychiatry 51, 199-214 (1994).

2. Kantrowitz, J. T. \& Javitt, D. C. N-methyl-d-aspartate (NMDA) receptor dysfunction or dysregulation: the final common pathway on the road to schizophrenia? Brain Res. Bull. 83, 108-121 (2010).

3. Moghaddam, B. \& Javitt, D. From revolution to evolution: the glutamate hypothesis of schizophrenia and its implication for treatment. Neuropsychopharmacology 37, 4-15 (2012).

4. Berman, R. M. et al. Antidepressant effects of ketamine in depressed patients. Biol. Psychiatry 47, 351-354 (2000).

5. Zarate, C. A. et al. A randomized trial of an N-methyl-D-aspartate antagonist in treatment-resistant major depression. Arch. Gen. Psychiatry 63, 856-864 (2006).

6. Miyamoto, S., Leipzig, J. N., Lieberman, J. A. \& Duncan, G. E. Effects of ketamine, MK-801, and amphetamine on regional brain 2-deoxyglucose uptake in freely moving mice. Neuropsychopharmacology 22, 400-412 (2000).

7. Moghaddam, B., Adams, B., Verma, A. \& Daly, D. Activation of glutamatergic neurotransmission by ketamine: a novel step in the pathway from NMDA receptor blockade to dopaminergic and cognitive disruptions associated with the prefrontal cortex. J. Neurosci. 17, 2921-2927 (1997).

8. Jackson, M. E., Homayoun, H. \& Moghaddam, B. NMDA receptor hypofunction produces concomitant firing rate potentiation and burst activity reduction in the prefrontal cortex. Proc. Natl Acad. Sci. USA 101, 8467-8472 (2004).

9. Lewis, D. A. \& Moghaddam, B. Cognitive dysfunction in schizophrenia: convergence of $\gamma$-aminobutyric acid and glutamate alterations. Arch. Neurol. 63, 1372-1376 (2006).

10. Lisman, J. E. et al. Circuit-based framework for understanding neurotransmitter and risk gene interactions in schizophrenia. Trends Neurosci. 31, 234-242 (2008).

11. Homayoun, H. \& Moghaddam, B. NMDA receptor hypofunction produces opposite effects on prefrontal cortex interneurons and pyramidal neurons. $J$. Neurosci. 27, 11496-11500 (2007).

12. Xu, X., Roby, K. D. \& Callaway, E. M. Immunochemical characterization of inhibitory mouse cortical neurons: three chemically distinct classes of inhibitory cells. J. Comp. Neurol. 518, 389-404 (2010).

13. Wang, Y. et al. Anatomical, physiological and molecular properties of Martinotti cells in the somatosensory cortex of the juvenile rat. J. Physiol. 561, 65-90 (2004).

14. Hakami, T. et al. NMDA receptor hypofunction leads to generalized and persistent aberrant $\gamma$ oscillations independent of hyperlocomotion and the state of consciousness. PLoS ONE 4, e6755 (2009).

15. Lütcke, H., Gerhard, F., Zenke, F., Gerstner, W. \& Helmchen, F. Inference of neuronal network spike dynamics and topology from calcium imaging data. Front. Neural Circuits 7, 201 (2013).

16. Kwan, A. C. \& Dan, Y. Dissection of cortical microcircuits by single-neuron stimulation in vivo. Curr. Biol. 22, 1459-1467 (2012).

17. Chen, T. W. et al. Ultrasensitive fluorescent proteins for imaging neuronal activity. Nature 499, 295-300 (2013).

18. Hamm, J. P., Peterka, D. S., Gogos, J. A. \& Yuste, R. Altered cortical ensembles in mouse models of schizophrenia. Neuron 94, 153-167. e158 (2017).

19. Koester, H. J. \& Sakmann, B. Calcium dynamics in single spines during coincident pre-and postsynaptic activity depend on relative timing of backpropagating action potentials and subthreshold excitatory postsynaptic potentials. Proc. Natl Acad. Sci. USA 95, 9596-9601 (1998).

20. Harris, J. A. et al. Hierarchical organization of cortical and thalamic connectivity. Nature 575, 195-202 (2019).

21. Wohleb, E. S. et al. GABA interneurons mediate the rapid antidepressant-like effects of scopolamine. J. Clin. Investig. 126, 2482-2494 (2016).

22. Ventura, A. et al. Cre-lox-regulated conditional RNA interference from transgenes. Proc. Natl Acad. Sci. USA 101, 10380-10385 (2004).

23. Tasic, B. et al. Adult mouse cortical cell taxonomy revealed by single cell transcriptomics. Nat. Neurosci. 19, 335-346 (2016).

24. Paul, A. et al. Transcriptional architecture of synaptic communication delineates GABAergic neuron identity. Cell 171, 522-539 (2017). e520.
25. Wang, H.-X. \& Gao, W.-J. Cell type-specific development of NMDA receptor in the interneurons of rat prefrontal cortex. Neuropsychopharmacology 34, 2028 (2009).

26. Gerhard, D. M. et al. GABA interneurons are the cellular trigger for ketamine's rapid antidepressant actions. J. Clin. Invest. https://doi.org/ 10.1172/JCI130808 (2019).

27. Han, C. J. et al. Trace but not delay fear conditioning requires attention and the anterior cingulate cortex. Proc. Natl Acad. Sci. USA 100, 13087-13092 (2003).

28. Swerdlow, N. R., Weber, M., Qu, Y., Light, G. A. \& Braff, D. L. Realistic expectations of prepulse inhibition in translational models for schizophrenia research. Psychopharmacology 199, 331-388 (2008).

29. Fanselow, M. S. From contextual fear to a dynamic view of memory systems Trends Cogn. Sci. 14, 7-15 (2010).

30. Armbruster, B. N., Li, X., Pausch, M. H., Herlitze, S. \& Roth, B. L. Evolving the lock to fit the key to create a family of $\mathrm{G}$ protein-coupled receptors potently activated by an inert ligand. Proc. Natl Acad. Sci. USA 104, 5163-5168 (2007).

31. Mateo, C., Knutsen, P. M., Tsai, P. S., Shih, A. Y. \& Kleinfeld, D. Entrainment of arteriole vasomotor fluctuations by neural activity is a basis of bloodoxygenation-level-dependent "resting-state" connectivity. Neuron 96, 936-948 (2017).

32. Widman, A. J. \& McMahon, L. L. Disinhibition of CA1 pyramidal cells by low-dose ketamine and other antagonists with rapid antidepressant efficacy. Proc. Natl Acad Sci. 115, 201718883 (2018).

33. Fan, L. Z. et al. All-optical synaptic electrophysiology probes mechanism of ketamine-induced disinhibition. Nat. Methods 15, 823 (2018).

34. Carlen, M. et al. A critical role for NMDA receptors in parvalbumin interneurons for gamma rhythm induction and behavior. Mol. Psychiatry 17, 537 (2012).

35. Belforte, J. E. et al. Postnatal NMDA receptor ablation in corticolimbic interneurons confers schizophrenia-like phenotypes. Nat. Neurosci. 13, 76 (2010).

36. Billingslea, E. N. et al. Parvalbumin cell ablation of NMDA-R1 causes increased resting network excitability with associated social and self-care deficits. Neuropsychopharmacology 39, 1603 (2014).

37. Tatard-Leitman, V. M. et al. Pyramidal cell selective ablation of N-methyl-Daspartate receptor 1 causes increase in cellular and network excitability. Biol. Psychiatry 77, 556-568 (2015).

38. Chiu, C. Q. et al. Compartmentalization of GABAergic inhibition by dendritic spines. Science 340, 759-762 (2013).

39. Marlin, J. J. \& Carter, A. G. GABA-A receptor inhibition of local calcium signaling in spines and dendrites. J. Neurosci. 34, 15898-15911 (2014).

40. Gentet, L. J. et al. Unique functional properties of somatostatin-expressing GABAergic neurons in mouse barrel cortex. Nat. Neurosci. 15, 607 (2012)

41. Villa, K. L. et al. Inhibitory synapses are repeatedly assembled and removed at persistent sites in vivo. Neuron 89, 756-769 (2016).

42. Kwon, T. et al. Ultrastructural, molecular and functional mapping of GABAergic synapses on dendritic spines and shafts of neocortical pyramidal neurons. Cereb. Cortex 29, 2771-2781 (2019).

43. Kim, Y. et al. Brain-wide maps reveal stereotyped cell-type-based cortical architecture and subcortical sexual dimorphism. Cell 171, 456-469. e422 (2017).

44. Demirtaş, M. et al. Hierarchical heterogeneity across human cortex shapes large-scale neural dynamics. Neuron 101, 1181-1194 (2019).

45. Barthas, F. \& Kwan, A. C. Secondary motor cortex: where 'sensory' meets 'motor' in the rodent frontal cortex. Trends Neurosci. 40, 181-193 (2017).

46. Yang, G. R., Murray, J. D. \& Wang, X.-J. A dendritic disinhibitory circuit mechanism for pathway-specific gating. Nat. Commun. 7, 12815 (2016).

47. Krystal, J. H. et al. Impaired tuning of neural ensembles and the pathophysiology of schizophrenia: a translational and computational neuroscience perspective. Biol. Psychiatry 81, 874-885 (2017).

48. Anticevic, A. et al. N-methyl-D-aspartate receptor antagonist effects on prefrontal cortical connectivity better model early than chronic schizophrenia. Biol. Psychiatry 77, 569-580 (2015).

49. Whitfield-Gabrieli, S. et al. Hyperactivity and hyperconnectivity of the default network in schizophrenia and in first-degree relatives of persons with schizophrenia. Proc. Natl Acad. Sci. USA 106, 1279-1284 (2009).

50. $\mathrm{Li}, \mathrm{N}$. et al. mTOR-dependent synapse formation underlies the rapid antidepressant effects of NMDA antagonists. Science 329, 959-964 (2010).

51. Phoumthipphavong, V., Barthas, F., Hassett, S., Kwan, A. C. Longitudinal effects of ketamine on dendritic architecture in vivo in the mouse medial frontal cortex. eNeuro 3, 0133-0115.2016 (2016).

52. Moda-Sava, R. et al. Sustained rescue of prefrontal circuit dysfunction by antidepressant-induced spine formation. Science 364, eaat8078 (2019).

53. Yuen, E. Y. et al. Repeated stress causes cognitive impairment by suppressing glutamate receptor expression and function in prefrontal cortex. Neuron 73, 962-977 (2012) 
54. Liston, C. et al. Stress-induced alterations in prefrontal cortical dendritic morphology predict selective impairments in perceptual attentional setshifting. J. Neurosci. 26, 7870-7874 (2006).

55. Holmes, S. E. et al. Lower synaptic density is associated with depression severity and network alterations. Nat. Commun. 10, 1529 (2019).

56. Hirsch, J. \& Crepel, F. Postsynaptic calcium is necessary for the induction of LTP and LTD of monosynaptic EPSPs in prefrontal neurons: an in vitro study in the rat. Synapse 10, 173-175 (1992).

57. Malenka, R. C., Kauer, J. A., Zucker, R. S. \& Nicoll, R. A. Postsynaptic calcium is sufficient for potentiation of hippocampal synaptic transmission. Science 242, 81-84 (1988).

58. Maeng, S. et al. Cellular mechanisms underlying the antidepressant effects of ketamine: role of $\alpha$-amino-3-hydroxy-5-methylisoxazole-4-propionic acid receptors. Biol. Psychiatry 63, 349-352 (2008).

59. Soumier, A. \& Sibille, E. Opposing effects of acute versus chronic blockade of frontal cortex somatostatin-positive inhibitory neurons on behavioral emotionality in mice. Neuropsychopharmacology 39, 2252 (2014).

60. Fino, E. \& Yuste, R. Dense inhibitory connectivity in neocortex. Neuron 69, 1188-1203 (2011).

61. Berger, T. K., Silberberg, G., Perin, R. \& Markram, H. Brief bursts self-inhibit and correlate the pyramidal network. PLoS Biol. 8, e1000473 (2010).

62. Taniguchi, H. et al. A resource of Cre driver lines for genetic targeting of GABAergic neurons in cerebral cortex. Neuron 71, 995-1013 (2011).

63. Franklin, K. B. J. \& Paxinos, G. The Mouse Brain In Stereotaxic Coordinates (Academic Press, 2007).

64. Lepack, A. E., Bang, E., Lee, B., Dwyer, J. M. \& Duman, R. S. Fast-acting antidepressants rapidly stimulate ERK signaling and BDNF release in primary neuronal cultures. Neuropharmacology 111, 242-252 (2016).

65. Chan, M.-H., Chiu, P.-H., Sou, J.-H. \& Chen, H.-H. Attenuation of ketamineevoked behavioral responses by mGluR5 positive modulators in mice. Psychopharmacology 198, 141-148 (2008).

66. Pologruto, T. A., Sabatini, B. L. \& Svoboda, K. ScanImage: flexible software for operating laser scanning microscopes. Biomed. Eng. Online 2, 13 (2003).

67. Pnevmatikakis, E. A. \& Giovannucci, A. NoRMCorre: an online algorithm for piecewise rigid motion correction of calcium imaging data. J. Neurosci. Methods 291, 83-94 (2017).

68. Petreanu, L. et al. Activity in motor-sensory projections reveals distributed coding in somatosensation. Nature 489, 299 (2012).

69. Bokil, H., Andrews, P., Kulkarni, J. E., Mehta, S. \& Mitra, P. P. Chronux: a platform for analyzing neural signals. J. Neurosci. Methods 192, 146-151 (2010).

70. Anagnostaras, S. G. et al. Automated assessment of pavlovian conditioned freezing and shock reactivity in mice using the video freeze system. Front. Behav. Neurosci. 4, 158 (2010).

\section{Acknowledgements}

We thank M. Picciotto for supplying pAAV-CMV-dsRed-pSico-GFP; S. Tomita, M. Higley, and J. Krystal for discussions, I.-J. Kim for use of microscope; V. Phoumthipphavong and M. Rapanelli for technical help. This work was supported by National
Institute of Mental Health grants R01MH112750 (A.C.K.) and R21MH110712 (A.C.K.), NARSAD Young Investigator Grant (A.C.K.), Simons Foundation Autism Research Initiative Pilot Award (A.C.K.), Inscopix DECODE Award (A.C.K.), Alzheimer's Association Research Fellowship AARF-17-504924 (F.A.), and James Hudson BrownAlexander Brown Coxe Postdoctoral Fellowship (F.A.).

\section{Author contributions}

F.A. and A.C.K. designed the research. F.A. performed surgery, imaging, electrophysiology, behavioral experiments, and histology. D.M.G., S.P., and R.S.D. performed molecular cloning, viral packaging, and in vitro validation. C.P. assisted with PPI. F.A., K.S., and A.C.K. analyzed all data. F.A. and A.C.K wrote the paper, with input from all other authors.

\section{Competing interests}

R.S.D. has consulted and/or received research support from Allergan, Naurex, Aptynix, Lilly, Johnson \& Johnson, Taisho, Relmada, and Navitor on unrelated projects. The remaining authors declare no competing interests.

\section{Additional information}

Supplementary information is available for this paper at https://doi.org/10.1038/s41467019-13809-8.

Correspondence and requests for materials should be addressed to A.C.K.

Peer review information Nature Communications thanks Eleanor Simpson, and the other, anonymous, reviewer(s) for their contribution to the peer review of this work. Peer reviewer reports are available.

Reprints and permission information is available at http://www.nature.com/reprints

Publisher's note Springer Nature remains neutral with regard to jurisdictional claims in published maps and institutional affiliations.

Open Access This article is licensed under a Creative Commons Attribution 4.0 International License, which permits use, sharing, adaptation, distribution and reproduction in any medium or format, as long as you give appropriate credit to the original author(s) and the source, provide a link to the Creative Commons license, and indicate if changes were made. The images or other third party material in this article are included in the article's Creative Commons license, unless indicated otherwise in a credit line to the material. If material is not included in the article's Creative Commons license and your intended use is not permitted by statutory regulation or exceeds the permitted use, you will need to obtain permission directly from the copyright holder. To view a copy of this license, visit http://creativecommons.org/ licenses/by/4.0/.

(C) The Author(s) 2020, corrected publication 2021 